\title{
On the (Non-)Equivalence of IRR and NPV
}

\author{
Thomas A. WEBER* \\ ${ }^{*}$ Chair of Operations, Economics and Strategy, Ecole Polytechnique Fédérale de Lausanne, \\ Station 5, CDM-ODY 1.01B, CH-1015 Lausanne, Switzerland. \\ E-mail: thomas.weber@epfl.ch. \\ MTEI Working Paper
}

October 2013

MTEI-WP-2013-001

\begin{abstract}
The internal rate of return (IRR) is generally considered inferior to the net present value (NPV) as a tool for evaluating and ranking projects, despite its inherently useful comparability to the cost of capital and the return of other investment opportunities. We introduce the Iselective IRR," a return criterion which, as a selection of an extended set of possible IRRs, is NPV-consistent. The selective IRR always exists, is unique, easy to compute, and does not suer from drawbacks that befall the project investment rate, the only other known NPVconsistent return criterion.
\end{abstract}

Keywords: Capital budgeting, internal rate of return, net present value.

JEL Classifications: C60, D01, E22, E40, G00, G10, G30, O16, O22. 


\title{
On the (Non-)Equivalence of IRR and NPV
}

\author{
Thomas A. Weber*
}

October 2013

\begin{abstract}
The internal rate of return (IRR) is generally considered inferior to the net present value (NPV) as a tool for evaluating and ranking projects, despite its inherently useful comparability to the cost of capital and the return of other investment opportunities. We introduce the "selective IRR," a return criterion which, as a selection of an extended set of possible IRRs, is NPV-consistent. The selective IRR always exists, is unique, easy to compute, and does not suffer from drawbacks that befall the project investment rate, the only other known NPV-consistent return criterion.
\end{abstract}

JEL-Classification: C60, D01, E22, E40, G00, G10, G30, O16, O22.

Keywords: Capital budgeting, internal rate of return, net present value.

${ }^{*}$ Chair of Operations, Economics and Strategy, École Polytechnique Fédérale de Lausanne, CDMODY 3.01, Station 5, CH-1015 Lausanne, Switzerland. Phone: +41 (0)21 69301 14. Fax: +41 (0)21 693 00 20. E-mail: thomas.weber@epfl.ch. 


\section{Introduction}

Despite the well-known shortcomings of the internal rate of return (IRR) as a criterion for evaluating the desirability of investment projects, it continues to be widely used in practice (Schall et al. 1978; Stanley and Block 1984; Graham and Harvey 2001; Brounen et al. 2004). Finance textbooks (e.g., Brealy and Myers 1991; Berk and DeMarzo 2007), on the other hand, usually suggest that selecting profitable investments amounts to undertaking projects with positive net present value (NPV). The greatest conceptual difficulty for the practically important IRR criterion is its failure to generally provide a unique value, against which to compare the company's hurdle rate in order to arrive at a decision that is consistent with the NPV rule (Samuelson 1937) 1 Multiple fixes have been proposed in the literature to guarantee existence and uniqueness, but usually they entail changing the underlying problem, e.g., by treating intermediate project cash flows differently according to their signs (resulting in the modified internal rate of return or MIRR; DuVillard 1787), by truncating the time horizon so as to maximize NPV (resulting in a different project; Arrow and Levhari 1969), or by changing the objective from value maximization to growth maximization (Dorfman 1981). All of them are inconsistent with the NPV rule.

In this paper, we propose a simple selective IRR criterion, which produces a unique number, consistent with both the definition of IRR (in the sense that it is equal to exactly one of its possible values) and the NPV rule. Our results are related to the project investment rate (PIR) proposed by Teichroew et al. (1965) which while producing NPV-consistent investment decisions is as a criterion generically different from any IRR and also suffers from a 'mixing inconsistency' (see Ex. 8). The selective IRR amounts to a single-valued selector defined on the set $\mathcal{I}$ of all possible (real-valued) IRRs, that (for any hurdle rate $r$ ) picks either the least element of $\mathcal{I}$ which exceeds $r$ when NPV is positive or the largest element of $\mathcal{I}$ smaller than $r$ when NPV is negative. Conceptually, the selective IRR is obtained as the limit of a tâtonnement process (in the form of a monotonic ratcheting procedure) that, starting with a given hurdle rate $r$, recursively produces a new PIR given the previous PIR as hurdle rate, thus converging to an element in $\mathcal{I}$ (or $\pm \infty$ ). When computing the selective IRR in this manner, the NPV is never directly used, so one obtains a complementary investment criterion (which produces NPVequivalent decisions). The selective IRR can be used in an incremental fashion to make pairwise NPV-consistent decisions between projects and thus to establish a preference order over a project portfolio.

In addition to resolving the long-standing divide between IRR and NPV, the selective IRR, which can be viewed as a piecewise constant function of $r$, provides an inbuilt robustness with respect to uncertainty in the hurdle rate. If a small random perturbation is added to the hurdle rate, then the selective IRR will most likely remain exact and deterministic, whereas the NPV generally becomes random. In addition, the use of the selective IRR as investment criterion fits the empirical observation that firms tend to

\footnotetext{
${ }^{1}$ The "hurdle rate" is defined here as the incremental cost of capital (or marginal cost of capital), that is the weighted average cost of capital for the additional capital required for the investment project under consideration (Downes and Goodman 1998, pp. 265/273). There are other uses of the term hurdle rate in the literature; see, e.g., Berk and DeMarzo (2007, pp. 738-739), Bodie et al. (1996, p. 247), or Dixit (1992).
} 
choose hurdle rates which exceed their true costs of capital (Poterba and Summers 1995). Indeed, firms can exaggerate their aspiration levels without effectively changing the selective IRR ${ }^{2}$ Doing this accounts (at least qualitatively) for some of the option value embedded in an investment opportunity: if the exaggeration of the hurdle rate triggers a jump in the selective IRR and thus a change in the investment decision, then, so the heuristic goes, the investment is no longer attractive because the net present value at the hurdle rate is not sufficiently positive to justify giving up the option of doing something else, such as simply delaying the project.

\section{$1.1 \quad$ Literature}

The notion that obtaining a cash flow in the future is worth less than obtaining it in the present can be traced back to discounting tables in the sixteenth century (Biondi 2006). Gioja (1817, p. 316) provides the classic form of the compound interest formula, determining the present value of a future cash flow by discounting it with an interest rate; he also provides the classic formula for the present value of an annuity (ibid., p. 319). The modern prominence of the NPV criterion as a basis for investment decisions is due to Fisher (1930). The NPV rule suffers from the drawback that by representing the net dollar value of a project the magnitude of this value cannot be informative about the rate of return, i.e., the amount gained for every invested dollar, which provides comparability with investments in capital markets. Other shortcomings of the NPV rule stem from its failure to include the value of various options embedded in a project (Ross 1995) or from encouraging misrepresentation in the presence of asymmetric information in an organization (Berkovitch and Israel 2004) $!^{3}$

The IRR, on the other hand, is implicit in the work of von Böhm-Bawerk (1889) who postulated an investment rule so as to maximize the net cash flow for each invested dollar. In its calculation "funds are assumed to earn, not the market rate of interest, but the rate of profit internal to the firm" (Scitovsky 1971, p. 214). Keynes (1936) terms the IRR therefore the marginal efficiency of capital 4 Lorie and Savage (1955) point out that the rate-of-return method is "ambiguous or anomalous" (p. 237) in the case where the NPV as a function of the discount rate $r$ has multiple roots, so the IRR (as the discount rate for which NPV vanishes) is not uniquely determined. One well-known attempt to modify or decompose the cash-flow stream so as to guarantee a unique rate of return, was introduced by Wright (1959), Flemming and Wright (1971), and independently by Arrow and Levhari (1969), all of whom require that there be "costless extricability" or in other words, that the project can be discontinued at any point in time, in which case the IRR is unique at the NPV-maximizing time horizon. But this IRR, although unique, provides

\footnotetext{
${ }^{2}$ Magni (2009) adds that the use of hurdle rates effectively deals with the bounded rationality of investors vis-à-vis the complexities of their investment decisions.

${ }^{3}$ For example, an option often embedded in an investment project is given by the ability to delay it, which invalidates the standard NPV criterion (invest when NPV is positive) because the opportunity cost of giving up the option by taking the investment decision is not considered (McDonald and Siegel 1986; Ingersoll and Ross 1992). In an organization, managers may try to hide high NPV projects if they entail less fixed capital than a project with lower (but still positive) NPV (Berkovich and Israel 2004).

${ }^{4}$ The marginal efficiency of capital is a special case of the "incremental IRR," that is the IRR of the difference of two cash-flow streams, also termed "rate of return over cost" by Fisher, when one of those cash-flow streams vanishes (Alchian 1955).
} 
only minimal information about the return of the original project and is generally not consistent with the NPV criterion (e.g., because it necessarily neglects any large capital expenditures at the end of a project). Hildreth (1946) and Dorfman (1981) point out that, under the additional assumption that projects are repeated indefinitely, selecting one with the largest real part of the IRR would maximize overall growth, the exact growth path being determined by all possible complex-valued IRR-values.5 This insight is achieved by modifying both the objective and the project (from finite-horizon to infinite-horizon), and therefore no significant connection to the NPV criterion can be expected.

The selective IRR in this paper is constructed using an idea by Teichroew et al. (1965) whose project investment rate is obtained by keeping track of the cash-on-hand (termed project balance) over time, until the end of the project horizon is reached. When compounding the project balance forward, the investor uses an external rate (usually the current hurdle rate) or an internal rate, depending on whether the current project balance is positive or negative, respectively. The project investment rate corresponds to the unique internal rate that renders the final project balance zero and thus presents the maximum load on negative project balances that the project can bear without turning into a net loss. The advantage of the project investment rate over the IRR is that it is unique and consistent with the NPV criterion. Its disadvantage is that it is different from any IRR and therefore does not inherit the desirable conceptual interpretation as marginal efficiency of capital, which is widely taught to practitioners of corporate finance (see, e.g., Brealey and Myers 1991) and enjoys widespread popularity in practice (Schall et al. 1978; Stanley and Block 1984; Graham and Harvey 2001). More seriously, the project investment rate leads to a mixing inconsistency in the sense that the convex combination of two positive-NPV projects with the same PIRs will generally have a larger PIR (see Prop. 2). Thus, if the NPVs of the two projects are the same, then two different owners could achieve a Pareto-improvement through cross-holdings. Our framework sidesteps this issue by requiring PIRs to be "in equilibrium," in the sense that the PIR remains unchanged when conditioning it on itself as the external rate; this in turn pinpoints (for this external hurdle rate) a unique "selective IRR" in the set of all possible IRRs.

More recently, two "product approaches" have been proposed, based on a product representation of the net present value $V(x, r)$ of the cash-flow stream $x$ at a given external rate $r$ in the form $V(x, r)=\alpha \cdot \beta$, where $\alpha=k-r$ is an excess return (of some internal rate $k$ relative to $r$ ) and $\beta=V(c, r)$ is the net present value of an associated investment stream $c$ at the rate $r$. Given a nonzero excess return $\alpha$ of any potential IRR-value $k$ (relative to $r$ ), Hazen (2003) shows how to determine an investment stream $c$ such that the product representation holds. Conversely, given any investment stream of nonzero NPV $\beta$, Magni (2010) shows how to find an excess return $\alpha$ such that the product representation holds. Both product approaches conclude that the NPV is positive if and only if $\alpha$ and $\beta$ have the same sign. More specifically, they proceed as follows: in order to compute the sign of $V(x, r)=\alpha \cdot \beta$, one of the factors (say, $\beta \neq 0$ ) is chosen in an essentially arbitrary manner (except that it be nonzero), which then implies the other factor (say, $\alpha=V(x, r) / \beta$; the latter amounts to computing the NPV of $x$ directly. Furthermore, due to the arbitrary nature of the choice of one factor, no additional information can be

${ }^{5}$ Pierru (2010) provides a (limited) interpretation of complex-valued solutions for the IRR in terms of returns of a two-asset portfolio; Osborne (2010) links the complex-valued solutions directly to the computation of a normalized NPV. 
obtained that is intrinsic to the evaluation of the investment project. Still, the product approaches remain useful for economic interpretation, e.g., when fixing one factor can be motivated by additional outside information ${ }^{6}$ Details about the product approaches in a continuous-time setting are provided in Appendix A, which also generalizes Hazen's (2003) result to any internal rate $k \neq r$ (as opposed to $k$ being limited to potential IRRvalues), which makes it clear that any excess return $\alpha \neq 0$ can be freely chosen together with a suitable investment stream that enables the product representation. The selective IRR introduced here provides intrinsic information about the return of the project. In particular, for any external rate $r$ there is a unique selective IRR $i(r)$. The corresponding excess return $\alpha=i(r)-r$ is such that $\alpha \cdot V(x, r) \geq 0$ always (with a strict inequality if and only if $V(x, r)>0)$. Hence, the selective IRR $i(r)$ is an $N P V$-consistent return criterion (see Appendix A), in the sense that the signs of the associated excess return $i(r)-r$ and of $V(x, r)$ are identical.

\subsection{Outline}

Sec. 2 introduces notation and basic concepts. Sec. 3 highlights the drawbacks of available return criteria for investment decisions, before Sec. 4 introduces the selective IRR and examines its properties. Sec. 5 concludes.

\section{Preliminaries}

Let $T>0$ be a fixed time horizon. Consider an investment project consisting of a deterministic cash-flow stream $x(t)$, which can also be represented by the (right-continuous) cumulative cash flow

$$
X(t)=\int_{0}^{t} x(s) d s
$$

for all $t \in[0, T]$. This setup enables a uniform treatment of discrete-time and continuoustime settings. $]^{7}$

Example 1 (Discrete Cash-Flow Stream). For positive integer T, a cash-flow stream with concentrated payments $x_{t}$ at the discrete times $t \in\{0,1, \ldots, T\}$ can be represented by the vector $\mathbf{x}=\left(x_{0}, \ldots, x_{T}\right)$, where $x_{0}=X(0)$ and $x_{t}=X(t)-X(t-1)$ for $t \in\{1,2, \ldots, T\} \mathrm{B}^{8}$ A discrete-time cash-flow stream is therefore fully specified by its vector of cash flows; this is used in the rest of paper.

\footnotetext{
${ }^{6}$ Magni (2010) introduces an "average IRR" (AIRR), $\bar{k}$, such that $\bar{k}=r+V(x, r) / \beta$ where $\beta=$ $V(c, r) \neq 0$ is the presentat value of a given investment stream; see Magni (2013) for a detailed comparison with the standard IRR. The flexibility in computing an excess return that responds to some observed investment stream (of interim project values) may be useful in certain applications such as real-estate projects where estimates of those interim values may be available (Altshuler and Magni, 2012).

${ }^{7}$ The cumulative cash flow $X$ is assumed to be a signed measure.

${ }^{8}$ In the general continuous-time framework, the discrete cash-flow stream corresponds to $x(t)=\mathbf{x}$. $\Delta_{T}(t)$, where $\Delta_{T}(t) \equiv(\delta(t), \delta(t-1), \ldots, \delta(t-T))$ has values of the same dimension, and $\delta(\cdot)$ denotes the Dirac distribution (Lang 1993, p. 298); it can be interpreted as a probability density with all its unit mass concentrated at the origin.
} 
Given an external rate $r$, representing a cost of capital or a hurdle rate 99 the present value of the cash-flow stream $x$ is

$$
V(x, r)=\int_{0}^{T} \exp (-r t) d X(t)
$$

Analogous to Teichroew et al. (1965) let the project balance $y(t, k, r), t \in[0, T]$, be the cumulative cash holdings under the cash-flow stream $x(t)$, provided that (i) $y\left(0^{-}, k, r\right)=0$ and (ii) compounding takes place at the external rate $r$ for positive holdings and at the internal rate $k$ for negative holdings. More precisely,

$$
y(t, k, r)=\int_{0}^{t}(f(y(s, k, r), k, r)+x(s)) d s, \quad \forall t \in[0, T],
$$

where

$$
f(y, k, r)= \begin{cases}k y, & \text { if } y<0, \\ r y, & \text { otherwise. }\end{cases}
$$

If the cash-flow stream $x$ is absolutely continuous, the integral equation (2) can be equivalently written as an initial-value problem,

$$
\left\{\begin{aligned}
\dot{y}(t, k, r) & =f(y(t, k, r), k, r)+x(t), \quad \forall t \in[0, T], \\
y\left(0^{-}, k, r\right) & =0,
\end{aligned}\right.
$$

and the corresponding project balance $y(\cdot, k, r)$ is continuous and differentiable almost everywhere. The project balance represents the cumulative free cash flow, that is the amount currently not invested in the project. When the project balance is negative, it indicates a net investment at that point. When the project balance is positive, it can be reinvested at the external rate.

Remark 1 (Continuous vs. Discrete Discounting). For discrete cash-flow streams, the discount factor $e^{-r t}$ is replaced by the discrete-time discount factor $1 /(1+\bar{r})^{t}$, where $\bar{r}=$ $e^{r}-1>-1$ is the discrete-time discount rate and $r \in \mathbb{R}$ is the unique corresponding continuous-time discount rate (with $r=\ln (1+\bar{r})$ ). ${ }^{10}$ The present value of the discrete cash-flow stream $x$, represented by the cash-flow vector $\mathbf{x}=\left(x_{0}, x_{1}, \ldots, x_{T}\right)$, is therefore

$$
V(x, r)=\sum_{t=0}^{T} \frac{x_{0}}{(1+\bar{r})^{t}} \equiv V[\mathbf{x}, \bar{r}] .
$$

Let $\mathbf{y}=\left(y_{0}, y_{1}, \ldots, y_{T}\right)$ denote the vector of the discrete-time project balance. Then

$$
\begin{aligned}
& y_{t}=f\left(y_{t-1}, 1+\bar{k}, 1+\bar{r}\right)+x_{t}, \quad t \in\{1,2, \ldots, T\}, \\
& y_{0}=x_{0} .
\end{aligned}
$$

All of the results in this paper apply directly to discrete cash-flow streams if one considers discrete time instances $t \in\{0,1, \ldots, T\}$ and $y(t, k, r)=y_{t}$ as defined in Eq. (2'). To connect to the extant literature and practical applications, discrete cash-flow streams are often used in our examples.

\footnotetext{
${ }^{9}$ Poterba and Summers (1995) observe that, to evaluate investment projects, firms frequently use hurdle rates which are quite different from their actual costs of capital.

${ }^{10}$ The only structural difference between continuous-time and discrete-time discount rates is that the latter are restricted to be greater than -1 whereas the former can take on any real values.
} 


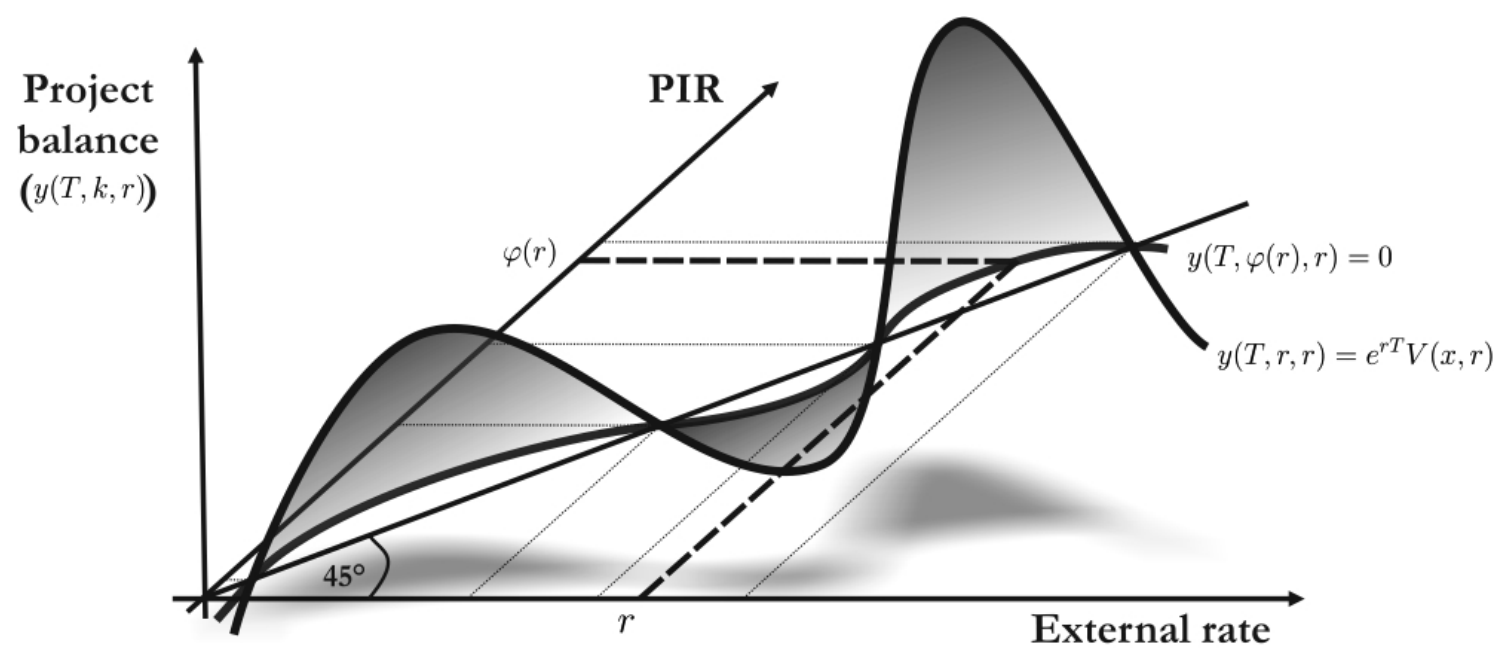

Figure 1: Relation between external rate, PIR, project balance, and present value.

Remark 2. Any measurable project balance $z(\cdot)=y(\cdot, k, r)$ can be generated by some cash-flow stream. Indeed, for a given $z$ and $(k, r)$ one obtains, by virtue of Eq. (2), the cumulative cash-flow measure $X(t) \equiv z(t)-\int_{0}^{t} f(z(s), k, r) d s$. If $z(\cdot)$ is differentiable, then $x(t) \equiv \dot{z}(t)-f(z(t), k, r)$ for all $t \in[0, T]$ is the corresponding cash-flow stream.

A change in the external or internal rate has a one-sided effect on the project balance.

Lemma 1. At any time $t \in[0, T]$, the project balance $y(t, k, r)$ is nonincreasing in $k$ and nondecreasing in $r$.

Proof. Since $f$ is nonincreasing in $k$ and nondecreasing in $r$, the monotonicity properties of $y$ follow directly from Eq. (2) for any fixed $t \in[0, T]$.

The project investment rate (PIR) of the cash-flow stream $x$ on $[0, T]$ is defined as ${ }^{11}$

$$
\varphi(r)=\sup \{\hat{k} \in \mathbb{R}: y(T, \hat{k}, r) \geq 0\},
$$

using the convention that $\sup \emptyset=-\infty$. Whenever $\varphi(r)$ is finite, necessarily

$$
y(T, \varphi(r), r)=0,
$$

a relation which can be used to determine the PIR in most interesting cases (see Fig. 1). The PIR is then the (maximum) internal rate which renders the project balance zero at the end of the horizon. At a finite $\varphi(r)$, the project is penalized for debt in a maximal way, such that at the end of the horizon it is as if the project had never taken place: there is no balance left to carry forward. From a formal viewpoint, one can define for any given internal rate $k$ a project financing rate (PFR) $\psi(k)$ in a dual manner,

$$
\psi(k)=\inf \{\hat{r} \in \mathbb{R}: y(T, k, \hat{r}) \geq 0\},
$$

\footnotetext{
${ }^{11}$ For notational convenience, the dependence on the cash-flow stream $x$ is dropped here and in similar definitions below. If important for the context (such as in La. 3), notation of the form $\varphi_{x}(r)$ emphasizes the dependence on $x$.
} 
where one sets inf $\emptyset=+\infty$. The PFR is the minimum external rate that, for a given internal rate, would guarantee the final project balance to be nonnegative. It is therefore the largest lower bound on the rate at which net gains of the project can be reinvested without the project turning into a loss at the end of its horizon. If $\psi(k)$ is finite, then necessarily

$$
y(T, k, \psi(k))=0
$$

so, comparing with Eq. (5), a finite PFR is in the preimage of any finite PIR and viceversa ${ }^{12}$ By the properties of the supremum and infimum of a set, the PIR exists and is unique as an element of $\overline{\mathbb{R}} \cdot 13$ Neither PIR nor PFR has to be finite.

Example 2. If $x(t) \equiv 0$, then $\varphi(r) \equiv \infty$ and $\psi(k) \equiv-\infty$. To obtain these PIR and PFR it is enough that $x(t)$ be nonnegative on $[0, T]$.

Corresponding to the monotonicity of the project balance in $(k, r)$ by La. 1 , the PIR and PFR must themselves be monotonic.

Lemma 2. The PIR $\varphi(\cdot)$ and the PFR $\psi(\cdot)$ are nondecreasing functions.

Proof. Fix two external rates, $\hat{r}$ and $r$, such that $\hat{r}>r$. Furthermore, let

$$
\mathcal{S}=\{\hat{k} \in \mathbb{R}: y(T, \hat{k}, r) \geq 0\} \quad \text { and } \quad \hat{\mathcal{S}}=\{\hat{k} \in \mathbb{R}: y(T, \hat{k}, \hat{r}) \geq 0\} .
$$

By La. 1, $y(T, k, \hat{r}) \geq y(T, k, r)$ for any given $k$ and $T$, which implies (using the same lemma) that $\mathcal{S} \subseteq \hat{\mathcal{S}}$. Thus, by Eq. (4), $\varphi(\hat{r})=\sup \hat{\mathcal{S}} \geq \sup \mathcal{S}=\varphi(r)$, which implies that the PIR is a nondecreasing function. The proof for the PFR proceeds in an analogous manner and is therefore omitted.

The monotonicity per se already implies a certain regularity, in that $\varphi(\cdot)$ and $\psi(\cdot)$ can have at most countably many discontinuities (Rudin 1976, pp. 91/100).

Remark 3. A (pure) net investment is such that

$$
y(t, k, r)=\int_{0}^{t} e^{k(t-s)} x(s) d s \leq 0
$$

for all $t \in[0, T]$. In that case, the PIR $k=\varphi(r)$ is independent of $r$ and can be determined uniquely by solving

$$
V(x, k)=\int_{0}^{T} e^{-k s} x(s) d s=0,
$$

provided that the situation is nontrivial such that $X \neq 0$. Similarly, a (pure) net financing is such that $y(t, k, r) \geq 0$ for all $t \in[0, T]$, and consequently $V(x, r)=0$ yields a unique and constant PFR $r=\psi(k)$, as long as $X \neq 0$.

\footnotetext{
${ }^{12}$ That is, $\psi(\varphi(r)) \in \varphi^{-1}(\varphi(r))$ and $\varphi(\psi(k)) \in \psi^{-1}(\psi(k))$ (see also the implicit function theorem, Rudin 1976, p. 224-225); neither $\varphi(\cdot)$ nor $\psi(\cdot)$ must be strictly monotonic, and if they are not, each being single-valued they cannot describe the same curve in $(k, r)$-space.

${ }^{13}$ For details on the extended field of real numbers, $\overline{\mathbb{R}}=[-\infty,+\infty]$, see, e.g., Aubin (1977, Sec. 1.3). In the discrete case (see Remark 1), the extended set of admissible discrete returns is $[-1, \infty]=\exp (\overline{\mathbb{R}})-1$.
} 
Example 3. Consider two discrete cash-flow streams by Dorfman (1981, p. 1012), represented by the cash-flow vectors $\mathbf{x}=(-1000,1100,0)$ and $\hat{\mathbf{x}}=(-1000,0,1166)$. Both are net investments; their discrete PIRs are $10 \%$ and approximately $8 \%$, respectively, independent of the external rate. On the other hand, the discrete PFR for any of these projects is -1 if the internal rate is less or equal than its PIR and $+\infty$ for $\bar{k} \geq 5.25$ 14

Example 4. The well-known discrete "pump project" (Lorie and Savage 1955, p. 237; Hirshleifer 1958, p. 349; Teichroew et al. 1965, p. 160) has a cash-flow vector of the form $\mathbf{x}=(-16,100,-100)$. It is neither a net-investment nor a net-financing project. As long as the PIR and the PFR are finite, they can be obtained from Eq. (2') in conjunction with (5) or (7), respectively, that is by solving

$$
y_{0}=-16, \quad y_{1}=-16(1+\bar{k})+100, \quad y_{2}=f\left(y_{1}, 1+\bar{k}, 1+\bar{r}\right)-100=0,
$$

whence $\varphi[\bar{r}]=(5.25 \bar{r}-1) /(1+\bar{r})$ for $\bar{r}>-1$; or equivalently: $\psi[\bar{k}]=(1+\bar{k}) /(5.25-\bar{k})$ for $-1<\bar{k}<5.25$ and $\psi[\bar{k}]=+\infty$ otherwise.

\section{Drawbacks of Extant Return Criteria}

\subsection{Internal Rate of Return}

The classical definition of internal rate of return (IRR) (or marginal efficiency of capital) of a cash-flow stream $x$ is as an external rate $i$ such that $V(x, i)=0$. Because this implicit definition guarantees neither the uniqueness nor the existence of an IRR, consider the set

$$
\mathcal{I}(x)=\{r \in \mathbb{R}: V(x, r)=0\}
$$

of all possible such rates ${ }^{15}$

Example 5. For cash-flow streams in Ex. 2 it is $\mathcal{I}(x)=\mathbb{R}($ when $c=0)$ or $\mathcal{I}(x)=\emptyset$ (when $c>0$ ). For the cash-flow stream in Ex. 4 it is $\mathcal{I}(x)=\{1 / 4,4\}$.

While the interpretation of a unique IRR as return is useful and widely accepted for assessing the marginal efficiency of an investment, even then it is not immediately clear why an investment criterion based on the comparison of IRRs should produce investment decisions that are consistent with the NPV criterion; see also La. 3 for a PIR-based approach to project selection.

Example 6. The IRRs for the discrete cash-flow streams in Ex. 3 are $10 \%$ for $\mathbf{x}$ and (about) $8 \%$ for $\hat{\mathbf{x}}$. On the other hand, at $\bar{r}=5 \%$ one obtains $V[\mathbf{x}, \bar{r}]<V[\hat{\mathbf{x}}, \bar{r}]$, whereas at $\bar{r}=10 \%$ it is $V[\mathbf{x}, \bar{r}]>V[\hat{\mathbf{x}}, \bar{r}]$.

The preceding example illustrates that to be compatible with NPV, any rate-of-return investment criterion (in addition to being uniquely determined) should depend on the external rate $r$ (see also Exs. 7 and 12 ).

\footnotetext{
${ }^{14}$ The discrete PIR is defined as $\varphi[\bar{r}]=\exp (\varphi(\ln (1+\bar{r})))-1$ for any discrete-time discount rate $\bar{r}>-1$, where $\bar{r}=e^{r}-1$ for any (continuous-time) discount rate $r \in \mathbb{R}$ (see Remark 1). Similarly, the discrete PFR is $\psi[\bar{k}]=\exp (\psi(\ln (1+\bar{k})))-1$ for all $\bar{k}>-1$. Hence, a PIR of $\varphi(r)=-\infty$ (resp., PFR of $\psi(k)=-\infty)$ corresponds to a discrete PIR of $\varphi[\bar{r}]=-1$ (resp., a discrete PFR of $\psi[\bar{k}]=-1$ ).

${ }^{15}$ For a discrete cash-flow stream $x$ with cash-flow vector $\mathbf{x}$ the set of discrete IRRs is given by $\mathcal{I}[\mathbf{x}]=$ $\{\bar{r}>-1: V[\mathbf{x}, \bar{r}]=0\}(=\exp (\mathcal{I}(x))-1)$, completely analogous to earlier definitions of equivalent discrete-time concepts (see Remark 1 and footnote 14 ).
} 


\subsection{Project Investment Rate}

Comparing the PIR $\varphi(r)$ to the external rate $r$ provides a sharp investment criterion, equivalent to the rule that the cash-flow stream $x$ is desirable at a given external rate $r$ if and only if $V(x, r) \geq 0$.

Proposition 1. For any external rate $r \in \mathbb{R}, V(x, r) \geq 0$ if and only if $\varphi(r) \geq r$. In addition, $V(x, r)=0$ if and only if $\varphi(r)=r$.

Proof. Fix an external rate $r$. Note first that by setting $k=r$, using the Cauchy formula (see, e.g., Weber 2011, Ch. 2), it is

$$
y(t, r, r)=\int_{0}^{t}(r y(s, r)+x(s)) d s=\int_{0}^{t} e^{r(t-s)} x(s) d s,
$$

which implies

$$
y(T, r, r)=e^{r T} V(x, r) .
$$

Assume now that $\varphi(r)$ is finite. Thus, if $V(x, r) \geq 0$, then by Eqs. (5) and (9), $y(T, r, r) \geq$ $0=y(T, \varphi(r), r)$ and therefore, using Las. 11 and 2, $\varphi(r) \geq r$. On the other hand, if $\varphi(r) \geq r$, then by Eq. (5) and La. 1, $y(T, \varphi(r), r)=0 \leq y(T, r, r)$. Hence, by Eq. (9) the present value $V(x, r) \geq 0$. If $\varphi(r)=\infty$, then $y(T, \hat{k}, r) \geq 0$ for any $\hat{k}$, so $(\operatorname{setting} \hat{k}=r)$ Eq. (9) again implies the nonnegativity of $V(x, r)$. Lastly, since $y(T, r, r)=e^{r T} V(x, r)$, by Eqs. (4)-(5): $V(x, r)=0$ is equivalent to $\varphi(r)=r$, concluding the proof.

Remark 4. One can formulate Prop. 1 equivalently in terms of the PFR, namely for any internal rate $k \in \mathbb{R}: V(x, k) \geq 0$ if and only if $\psi(k) \leq k$. We focus attention attention on PIR as an investment criterion, as all results involving the PIR have an equivalent formulation in terms of the PFR.

Remark 5. Prop. 1 (in conjunction with Remark 4) can be compactly stated as follows: $(\varphi(r)-r) V(x, r)>0$ for all $r \notin \mathcal{I}(x)$ (resp., $(\psi(k)-k) V(x, k)<0$ for all $k \notin \mathcal{I}(x))$. The result implies in particular that $V(x, r)>0 \Leftrightarrow \varphi(r)>r(\operatorname{resp} ., V(x, k) \Leftrightarrow \psi(k)<k)$.

The decision between two mutually exclusive investment projects, $x$ and $\hat{x}$, needs to be taken based on the return $\varphi_{\hat{x}-x}$ (incremental PIR) of the difference of the two corresponding cash-flow streams ${ }^{16}$ The idea is to evaluate the profitability of $\hat{x}-x$, corresponding to an upgrade from $x$ to $\hat{x}$, since $\hat{x}=x+(\hat{x}-x)$.

Lemma 3. Let $x(t)$ and $\hat{x}(t)$, for $t \in[0, T]$, be two cash-flow streams, and let $\varphi_{\hat{x}-x}(r)$ denote the return of the differential cash-flow stream, $\hat{x}-x$. Then $V(\hat{x}, r) \geq V(x, r)$ if and only if the incremental PIR $\varphi_{\hat{x}-x}(r) \geq r$.

Proof. The claim follows immediately from the fact that $V(\hat{x}, r) \geq V(x, r)$ if and only if $V(\hat{x}-x, r) \geq 0$.

\footnotetext{
${ }^{16}$ Without loss of generality one can restrict attention to the pairwise comparison of mutually exclusive cash-flow streams. Arbitrary dependencies between cash-flow streams can be accommodated by defining each possible choice and resulting cash-flow stream as an "alternative" that, by construction, is mutually exclusive to any other such alternative.
} 


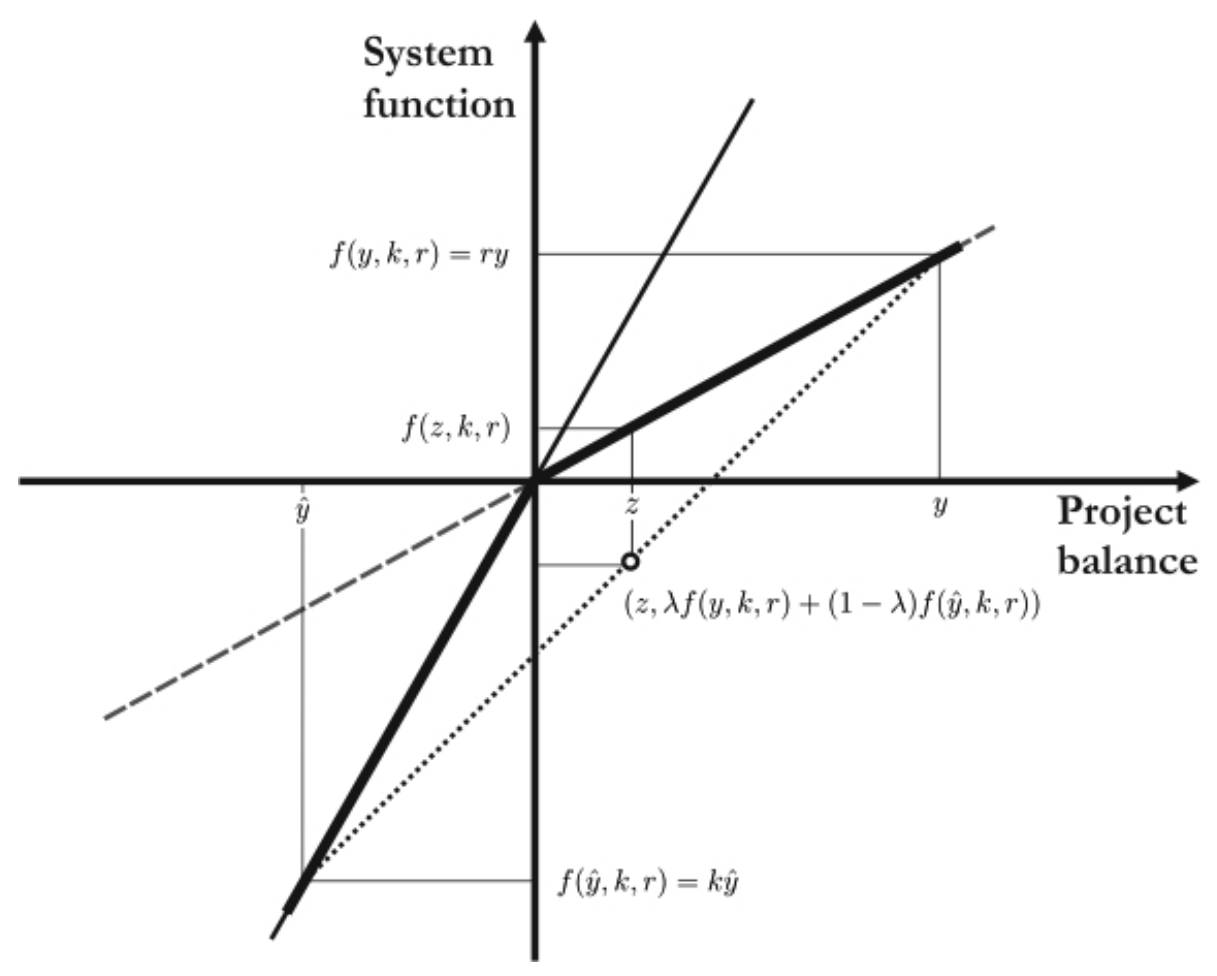

Figure 2: Concavity of the system function $f(\cdot, k, r)$ for $k \geq r$.

Example 7. The (discrete) PIR of the difference of the discrete cash-flow streams in Ex. 3 is $\varphi_{\hat{x}-x}[\bar{r}] \equiv(1166 / 1100)-1=6 \%$, so $\hat{x}$ is preferable as investment (consistent with the NPV criterion) if and only if the incremental PIR of $6 \%$ exceeds the external rate $\bar{r} \cdot 17$

The PIR always exists, is unique, and depends on the external rate. Most importantly, it is compatible with the NPV criterion for investment decisions. Yet it suffers from the drawback that it endorses the mixing of profitable (i.e., positive-NPV) projects and tends to reject the mixing of unprofitable projects. This is clarified by the following result.

Proposition 2. Let $x(t)$ and $\hat{x}(t)$, for $t \in[0, T]$, be two cash-flow streams with identical PIR $\varphi(r)$ at a given external rate $r$. If $\varphi_{\lambda}(r)$, for $\lambda \in[0,1]$, denotes the PIR of the cash-flow stream $\lambda x+(1-\lambda) x$, then $(\varphi(r)-r)\left(\varphi_{\lambda}(r)-\varphi(r)\right) \geq 0$.

Proof. Let $y(t, k, r)$ (resp., $\hat{y}(t, k, r))$ be the project balance for the cash-flow stream $x(t)$ (resp., $\hat{x}(t))$ on $[0, T]$ with PIR $k=\varphi(r)$ (so $y(T, k, r)=0)$, and fix $\lambda \in[0,1]$. Both $y$ and $\hat{y}$ satisfy initial-value problems of the form (3) ${ }^{18}$ Similarly, the project balance $y$ of

\footnotetext{
${ }^{17}$ It is important that $x$ and $\hat{x}$ be compared over the same time horizon T. Alluding to Hildreth (1946), Dorfman (1981) pointed out that this may not be clear a priori. For example, for the cash-flow vector $\mathbf{x}=$ $(-1000,1100,0)$ it might be possible to reinvest the project balance at $t=1$ at the same return $\bar{k}=10 \%$, that would result in the cash-flow vector $\mathbf{x}^{\prime}=(-1000,0,1210)$ which is strictly greater than $\hat{\mathbf{x}}$, so by Ex. 9 the incremental selective IRR becomes $+\infty$ (meaning that $\mathbf{x}^{\prime}$ is strictly better than $\hat{\mathbf{x}}$ ), despite the fact that the selective IRR corresponding to $\mathbf{x}^{\prime}$ remains the same as the selective IRR corresponding to $\mathbf{x}$.

${ }^{18}$ To simplify the presentation, the initial-value problem (3) is used instead of the integral equation (2); if the cash-flow stream contains atoms (such as in the discrete case; see Ex. 1) the former is still valid when interpreted in the context of the theory of distributions (Schwartz 1950).
} 
the cash-flow stream $\lambda x+(1-\lambda) \hat{x}$ on $[0, T]$ satisfies the initial-value problem

$$
\dot{y}=f(y, k, r)+\lambda x+(1-\lambda) \hat{x}, \quad y\left(0^{-}, k, r\right)=0 .
$$

On the other hand, setting $z=\lambda y+(1-\lambda) \hat{y}$ by the initial-value problem (3),

$$
\dot{z}=\lambda f(y, k, r)+(1-\lambda) f(\hat{y}, k, r)+\lambda x+(1-\lambda) \hat{x}, \quad z\left(0^{-}, k, r\right)=0 .
$$

Rewriting the system function $f$ in the form

$$
f(y, k, r)= \begin{cases}\min \{k y, r y\}, & \text { if } k \geq r, \\ \max \{k y, r y\}, & \text { otherwise }\end{cases}
$$

it becomes apparent that $f(\cdot, k, r)$ is concave for $k \geq r$ (see Fig. 2) and convex for $k \leq r$. Hence,

$$
\dot{z} \geq f(z, k, r)+\lambda x+(1-\lambda) \hat{x}, \quad \text { for } k \geq r
$$

and

$$
\dot{z} \leq f(z, k, r)+\lambda x+(1-\lambda) \hat{x}, \quad \text { for } k \leq r .
$$

$\mathrm{C}$ By virtue of the identical initial conditions, $y\left(0^{-}, k, r\right)=z\left(0^{-}, k, r\right)=0$, therefore $y$ exceeds $z$ at least weakly for $k \geq r$, and vice-versa for $k \leq r$. That is $(k-r)(y-z) \geq 0$, and in particular

$$
(k-r)(y(T, k, r)-z(T, k, r)) \geq 0 .
$$

Hence, La. 1 and Eq. (4) together imply that the PIR of $\lambda x+(1-\lambda) \hat{x}$, denoted by $\varphi_{\lambda}(r)$, is greater than (resp., less than) $k=\varphi(r)$ for $k \geq r$ (resp., $k \leq r$ ).

Prop. 2 states that two profitable cash-flow streams with the same return appear even more profitable in terms of their returns if they are mixed. For unprofitable projects the converse obtains. The following example illustrates this fact.

Example 8 (Mixing Inconsistency). Let $\bar{r}=10 \%$ be a given (discrete-time) external rate. Consider two discrete cash-flow streams over the horizon $T=2$, described by the cashflow vectors $\mathbf{x}=(-1100,-13200,17424)$ and $\hat{\mathbf{x}}=(-14300,18000,-924)$, respectively. Their present values both equal 1300 and their discrete PIRs are both equal to 20\%. The convex combination of these cash flows, described by $\mathbf{x}_{\lambda}=\lambda \mathbf{x}+(1-\lambda) \hat{\mathbf{x}}$, for $\lambda \in[0,1]$, leaves the present value unchanged, but its discrete PIR $\varphi_{\lambda}[\bar{r}]$ exceeds $\varphi_{0}[\bar{r}]=\varphi_{1}[\bar{r}]=20 \%$ for $\lambda \in(0,1)$; see Fig. 3 .

A consequence of the last example is that a firm interested in maximizing the return for its shareholders measured as PIR might be able to do so by engaging in a leveraged buyout of a target that is inferior in terms of both return and present value. To see this, it is enough to slightly decrease the present value and return of one of the projects in Ex. 8 , which at $\lambda=0.5$ (because of the continuity of all measures in $\lambda$ ) would still yield a larger PIR than each individual project by itself. The apparent Pareto-improvement from the mixing of projects is inconsistent with the notion of an unbiased performance measure. 


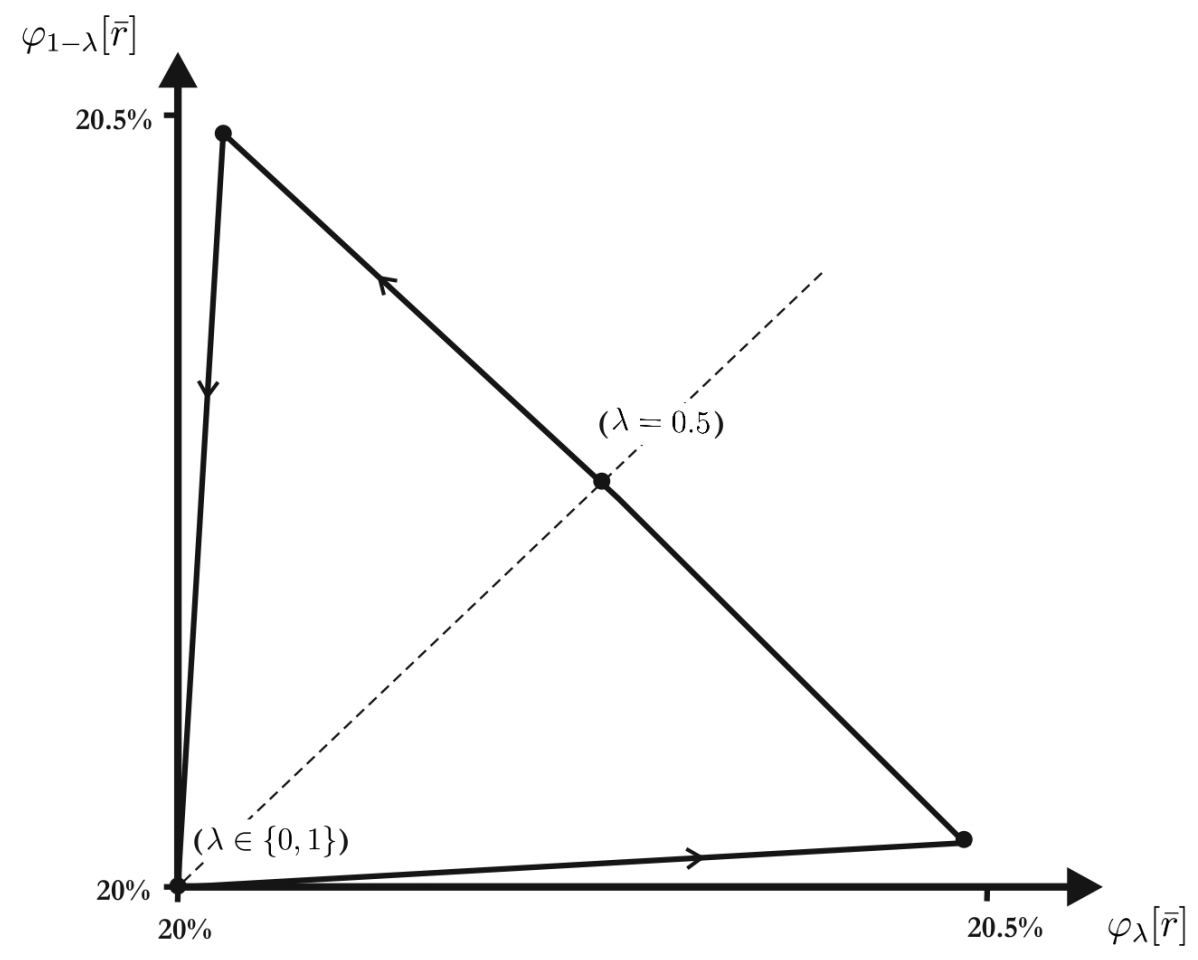

Figure 3: Mixing inconsistency of the project investment rate in Ex. 8 ,

\section{Selective IRR as Investment Criterion}

The idea for a return-based investment criterion that combines the advantages of IRR (interpretation, historical significance, widespread use) and PIR (existence, uniqueness, dependence on external rate) is based on an internal consistency that is achieved by repeatedly ratcheting up (or down) a given initial external hurdle rate to a new rate, which then becomes itself the external rate at which to evaluate a given investment project. To arrive at the definition of a "selective IRR" as a consistent investment criterion, it is useful to first bracket all reasonable rates of return for a project, given any external rate $r$.

Let $i_{-}(r)=\sup \{\hat{r} \in(-\infty, r]: V(x, \hat{r})=0\}$ and $i_{+}(r)=\inf \{\hat{r} \in[r, \infty): V(x, \hat{r})=0\}$, where as before $\sup \emptyset=-\infty$ and $\inf \emptyset=+\infty$. Now define the selective IRR as

$$
i(r)= \begin{cases}i_{+}(r), & \text { if } \varphi(r) \geq r \\ i_{-}(r), & \text { otherwise }\end{cases}
$$

From Eq. (8), both $i_{+}(r)$ and $i_{-}(r)$ lie in the extended set of IRRs,

$$
\overline{\mathcal{I}}(x)=\mathcal{I}(x) \cup\{-\infty,+\infty\}
$$

moreover, these "bracket rates" bound the PIR. The two additional elements, $-\infty$ and $\infty$, in the extended set of IRRs $\overline{\mathcal{I}}(x)$ are important to guarantee the existence of a selective IRR. The extra elements also make perfect sense as illustrated in the next example.

Example 9. (i) If, as in Ex. 2, $x(t) \geq 0$ for all $t \in[0, T]$, then $i(r) \equiv+\infty$. (ii) If $x(t)<0$ 
for all $t \in[0, T]$, then $i(r) \equiv-\infty$. (iii) If $x(t)=t-1$, for all $t \in[0, T]$, and $T=2$, then

$$
i(r)= \begin{cases}+\infty, & \text { if } r<0 \\ 0, & \text { if } r=0 \\ -\infty, & \text { if } r>0\end{cases}
$$

An unbounded selective IRR of $i(r)=\infty$ for a given $r$ is by construction equivalent to $i_{+}(r)=\infty$ (by Eq. (10)) and $V(x, r)>0$ (by Prop. 1). The latter means that increasing the external rate beyond $r$ cannot decrease the present value of the project to zero, i.e., for any $\hat{r} \geq r$ it is $V(x, \hat{r})>0$. Similarly, if $i(r)=-\infty$, then $V(x, \hat{r})<0$ for all $\hat{r} \leq r$.

Remark 6 (Discrete Selective IRR). For a discrete cash-flow stream $x$ with cash-flow vector $\mathbf{x}$ (see Remark 1) the extended set of discrete IRRs is

$$
\overline{\mathcal{I}}[\mathbf{x}]=\mathcal{I}[\mathbf{x}] \cup\{-1, \infty\}
$$

where $\mathcal{I}[\mathbf{x}]=\{\bar{r}>-1: V[\mathbf{x}, \bar{r}]=0\}$ is the set of discrete IRRs (see footnote 15). With the discrete bracket rates $i_{ \pm}[\bar{r}]=\exp \left(i_{ \pm}(\ln (1+\bar{r}))\right)-1$ the discrete selective IRR becomes

$$
i[\bar{r}]= \begin{cases}i_{+}[\bar{r}], & \text { if } \varphi[\bar{r}] \geq \bar{r}, \\ i_{-}[\bar{r}], & \text { otherwise }\end{cases}
$$

for all $\bar{r}>-1$, where $\varphi[\bar{r}]=\exp (\varphi(\ln (1+\bar{r})))-1$ is the discrete PIR of $x$ (see footnote 14).

Lemma 4. For any external rate $r \in \mathbb{R}$, it is $i_{-}(r) \leq \varphi(r) \leq i_{+}(r)$.

Proof. Begin with the second inequality. By Prop. 1.

$$
\{\hat{r} \in[r, \infty): V(x, \hat{r})=0\}=\{\hat{r} \in[r, \infty): \varphi(\hat{r})=\hat{r}\} .
$$

If $i_{+}(r)$ is infinite, there is nothing to show. If $i_{+}(r)<\infty$, then there exists a finite number $\hat{r}$ such that $i_{+}(r)=\varphi(\hat{r})=\hat{r} \geq r$. Thus, by La. 2, $\varphi(r) \leq \varphi(\hat{r})=i_{+}(r)$, which is the second inequality. The first inequality follows from a symmetric argument.

We are now ready for a recursive construction of the selective IRR, as a limit of ratcheted hurdle rates. This construction is independent of Eq. (10), but it leads to the same result. The key for establishing convergence is the monotonicity and boundedness of the resulting sequence of returns.

Proposition 3. For any external rate $r \in \mathbb{R}$, it is $\varphi(\varphi(\varphi(\cdots \varphi(r) \cdots))) \rightarrow i(r)$.

Proof. We distinguish three cases, based on the sign of $V(x, r)$. 1. If $V(x, r)>0$, then by Prop. 1 it is $\varphi(r)>r$. Consider now the sequence $\sigma=\left(r_{n}\right)_{n=0}^{\infty}$ with $r_{0}=r$ and $r_{n+1}=$ $\varphi\left(r_{n}\right)$ for all $n \geq 0$. Then by assumption, $r_{1} \geq r_{0}$. Furthermore, if $r_{n+1} \geq r_{n}$, then using the monotonicity of $\varphi(\cdot)$ (by La. 2), $r_{n+2}=\varphi\left(r_{n+1}\right) \geq \varphi\left(r_{n}\right)=r_{n+1}$. Thus by induction, $r_{n+1} \geq r_{n}$ for all $n \geq 0$. It is well known (see, e.g., Rudin 1976, Thm. 3.14) that the monotonic sequence $\sigma$ converges if and only if it is bounded. By definition of $i_{+}(\cdot)$ and Eq. (12), $i_{+}\left(r_{0}\right)$ (which is equal to $i(r)$ because $r_{0}=r$; furthermore $i_{+}(r) \geq r$ ) is the largest lower bound of the set $\mathcal{S}(r)=\{\hat{r} \in[r, \infty): \varphi(\hat{r})=\hat{r}\}$, which must also be an upper bound for $\sigma$, and $\sigma$ therefore has a limit, $r_{\infty} \leq i_{+}(r)$. Thus, necessarily $\varphi\left(r_{\infty}\right)=r_{\infty}$, 


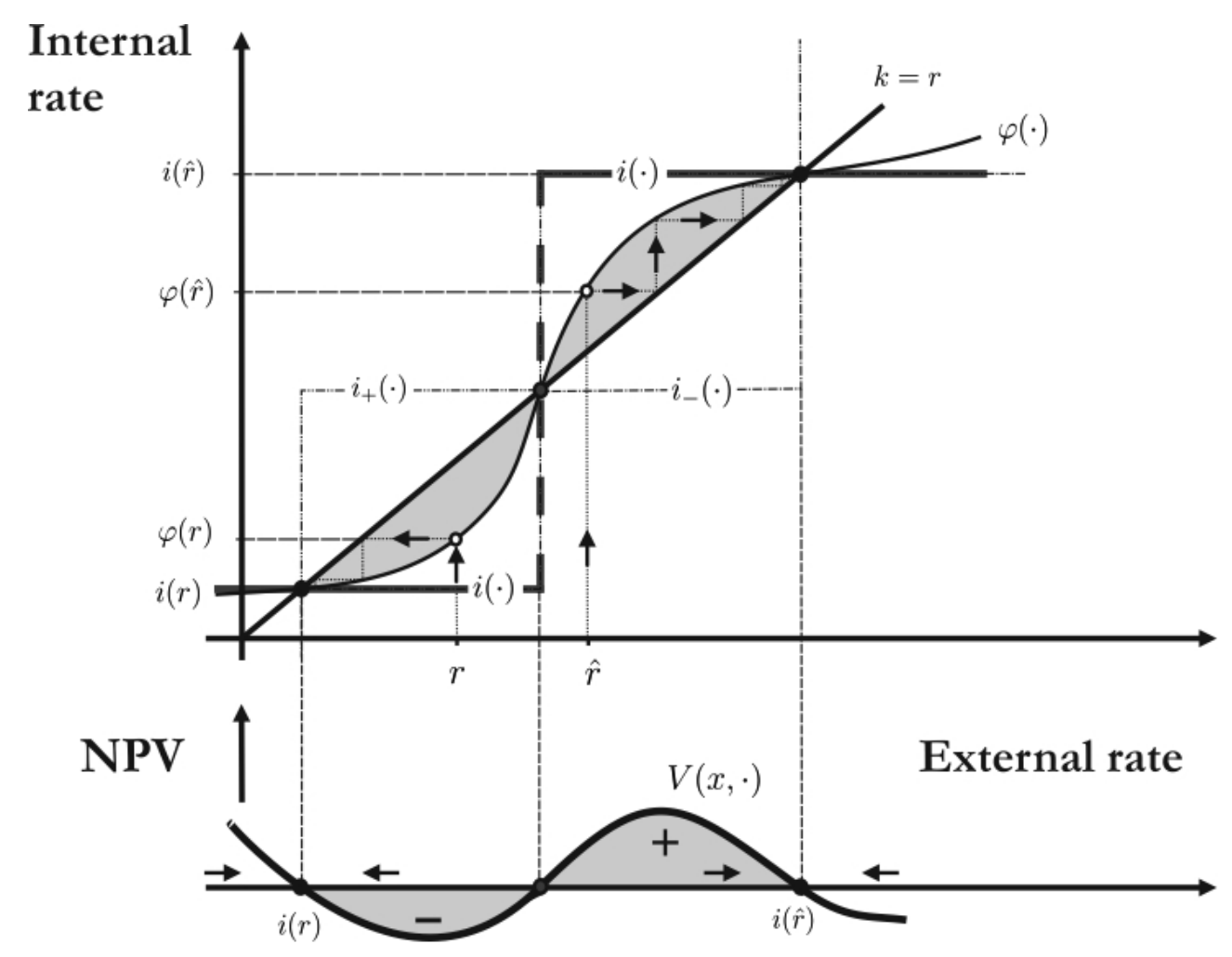

Figure 4: Computation of the selective IRR.

since otherwise $r_{\infty}$ could not have been the limit of $\sigma$. But this means $r_{\infty}$ is an element of the set $\mathcal{S}(r)$, which in turn implies that $r_{\infty}=\inf \mathcal{S}(r)=i_{+}(r)$. 2. If $V(x, r)=0$, then the claim follows trivially because $r=\varphi(r)=i_{+}(r)=i_{-}(r)$. 3. If $V(x, r)<0$, then the claim follows using arguments that are symmetric to the case where $V(x, r)>0$. This completes the proof.

Fig. 4 illustrates the recursive ratcheting procedure. The last result implies the fixedpoint relation

$$
\varphi(i(r))=i(r),
$$

because $V(x, \hat{r})=0$ if and only if $\varphi(\hat{r})=\hat{r}$; furthermore, $V(x, i(r))=0$ by definition of $i(r)$. Thus, the selective IRR $i(r)$ is a fixed point of the PIR map $\varphi(\cdot)$. The fixed point is stable, i.e., can appear as a limit of a ratcheting procedure that starts elsewhere, if $(i(r)-i)(\varphi(i)-i)>0$ for all $i \neq i(r)$ in some neighborhood of $i(r)$ 19 Fig. 4 shows the stable fixed points $i(r), i(\hat{r})$ and the unstable fixed point where $i(\cdot)$ jumps.

Example 10. Consider again the "pump project" of Ex. 4. The solution set of the fixed-point problem $\varphi[\bar{r}]=\bar{r}$ is $\mathcal{I}[\mathbf{x}]=\{1 / 4,4\}$. For $\bar{r}=1 / 7<1 / 4$, one obtains $\varphi[\bar{r}]=$ $-7 / 32<\bar{r}$, so by the recursive ratcheting procedure, $i[\bar{r}]=-\infty$. On the other hand, for $\bar{r}=1 / 3$ in $(1 / 4,4]$, one obtains $\varphi[\bar{r}]=9 / 16>\bar{r}$ and therefore $i[\bar{r}]=4$. More generally,

$$
i[\bar{r}]= \begin{cases}-1, & \text { if } \bar{r} \in(-1,1 / 4), \\ 1 / 4, & \text { if } \bar{r}=1 / 4, \\ 4, & \text { if } \bar{r}>1 / 4,\end{cases}
$$

\footnotetext{
${ }^{19}$ If $\varphi(\cdot)$ is differentiable, then the fixed point $i(r)$ is stable if $\dot{\varphi}(i(r))<1$; it is unstable if $\dot{\varphi}(i(r))>1$.
} 
for any external rate $\bar{r}>-1$.

Remark 7 (Numerical Computation). In practice, finding the selective IRR $i(r)$ for a given cash-flow stream $x$ at the rate $r$ is simpler than determining $\mathcal{I}(x)$, because instead of determining all roots $k$ of $V(x, k)$ it merely requires finding the next zero of the NPV in a known direction of the external rate, ${ }^{20}$ depending on the sign of $V(x, r)$, at the given external rate $r{ }^{21}$ If $V(x, r)>0$ (resp., $\left.V(x, r)<0\right)$, then $i(r)$ is the closest element in $\overline{\mathcal{I}}(x)$ to the right (resp., left) of $r$.

The monotonicity properties of the PIR are inherited by the selective IRR.

Lemma 5. The selective IRR $i(\cdot)$ is a nondecreasing function.

Proof. Both $i_{-}(\cdot)$ and $i_{+}(\cdot)$ are nondecreasing functions, so $i(\cdot)$ is by Eq. (10), as composition of nondecreasing functions, also a nondecreasing function.

The usefulness of the selective IRR as an investment criterion rests on the following result, which establishes that the external rate and the selective IRR necessarily bracket the PIR.

Lemma 6. For any external rate $r \in \mathbb{R}$, the $P I R \varphi(r)$ lies between $r$ and the selective IRR $i(r)$, that is $(\varphi(r)-r)(i(r)-\varphi(r)) \geq 0$.

Proof. If $\varphi(r) \geq r$, then by the proof of Prop. 3, $i(r)=i_{+}(r) \geq \varphi(r) \geq r$. Similarly, if $\varphi(r) \leq r$, then $i(r)=i_{-}(r) \leq \varphi(r) \leq r$. Combining both conclusions yields the inequality in the lemma for any given external rate $r$.

By virtue of La. 6, $\varphi(r)=r$ if and only if $i(r)=\varphi(r)$. In other words, all the fixed points of $\varphi(\cdot)$ are also fixed points of $i(\cdot)$; Prop. 1 implies that the NPV must therefore vanish exactly at those fixed points. This together with the preceding lemma is enough to establish the selective IRR as equivalent to the NPV criterion.

Proposition 4. For any external rate $r \in \mathbb{R}, V(x, r) \geq 0$ if and only if $i(r) \geq r$. In addition, $V(x, r)=0$ if and only if $i(r)=r$.

Proof. By La. 6, $i(r) \geq r$ implies that $\varphi(r) \geq r$. Conversely, if $\varphi(r) \geq r$, then by the proof of Prop. 3, $i(r)=i_{+}(r) \geq \varphi(r) \geq r$. Hence, $(i(r)-r)(\varphi(r)-r) \geq 0$ for all $r$. Lastly, by Prop. $1 V(x, r)=0$ is equivalent to $\varphi(r)=r$, which by Prop. 3 implies that $i(r)=r$. Conversely, if $i(r)=r$, then $r \in \mathcal{I}(x)$ and thus $V(x, r)=0$, completing the proof.

The selective IRR works as investment criterion also in the somewhat pathological case where $V(x, r)$ is nonpositive for all $r$ and $V(x, r)=0$ for some $r$, shown below.

\footnotetext{
${ }^{20}$ One may use the well-known Pegasus method, an efficient variation of the Newton gradient method, which avoids direct computation of the gradient (Dowell and Jarratt 1972).

${ }^{21}$ The implied partition is reminiscent of the method proposed by Hartman and Schafrick (2004), which splits the graph of $V(\cdot, r)$ horizontally according to the sign of its first derivative. Yet their method does not yield a unique "relevant" IRR when $r$ coincides with a local extremum of $V$, i.e., when $\left.\frac{\partial V}{\partial x}\right|_{(x, r)}=0$.
} 
Example 11 (Hartman and Schafrick 2004, Ex. 2). Let $x$ be a discrete cash-flow stream with cash-flow vector $\mathbf{x}=(-1,4,-4)$. Then $\overline{\mathcal{I}}[\mathbf{x}]=\{-1,1, \infty\}$ and the discrete PIR is $\varphi[\bar{r}]=(3 \bar{r}-1) /(1+\bar{r})$ for all $\bar{r}>-1$. The only fixed point of $\varphi[\cdot]$ is 1 (i.e., $\varphi[1]=1)$. The selective IRR becomes

$$
i[\bar{r}]= \begin{cases}-1, & \text { if } \bar{r} \in(-1,1), \\ 1, & \text { if } \bar{r} \geq 1 .\end{cases}
$$

Hence, $i[\bar{r}]<\bar{r}$ for all $\bar{r} \in(-1, \infty) \backslash\{1\}$ and $i[1]=1$. By Prop. 4 it is therefore $V[\mathbf{x}, 1]=0$ and $V[\mathbf{x}, \bar{r}]<0$ for $\bar{r} \in(-1, \infty) \backslash\{1\}$.

It is clear that the notion of an "incremental selective IRR," defined as the selective IRR of the difference of two cash-flow streams, can be used to decide between two mutually exclusive investment projects, in analogy to La. 3 .

Example 12. Just like the incremental PIR in Ex. 7 the selective IRR can be applied "incrementally" to the difference of the discrete cash-flow streams in Ex. 3. One obtains $i_{\hat{x}-x}[\bar{r}] \equiv 6 \%$. Thus, $\hat{x}$ is preferred over $x$ if and only if $i_{\hat{x}-x}[\bar{r}] \geq \bar{r}$, which holds if and only if $-1<\bar{r} \leq 6 \%$.

The selective IRR cannot produce an inconsistency in the sense of Prop. 2 when combining different projects with the same return. The fundamental reason is that the intersection of the sets of IRR values for two different cash-flow streams $x$ and $\hat{x}$ stay IRR values of any linear combination of these cash-flow streams; more specifically,

$$
\mathcal{I}(x) \cap \mathcal{I}(\hat{x}) \subseteq \mathcal{I}(\mu x+\hat{\mu} \hat{x})
$$

for any $\mu, \hat{\mu}$ in $\mathbb{R}$.

Example 13. The mixing inconsistency in Ex. 8 does not appear when evaluating the mixed cash-flow stream using the selective IRR. Denoting the latter $i_{\lambda}[\bar{r}]$, it in fact does not depend on $\lambda \in[0,1]$, which follows directly from Eq. (14) with $(\mu, \hat{\mu})=(\lambda, 1-\lambda)$.

Remark 8 (Intrinsic External Rate). Analogous to PIR vs. PFR (see Remark 4), for a given internal rate $k$ there is, analogous to the selective IRR, the "intrinsic external rate" $j(k)$ is defined as

$$
j(k)= \begin{cases}j_{+}(k), & \text { if } \psi(k) \geq k \\ j_{-}(k), & \text { otherwise }\end{cases}
$$

where $j_{-}(k)=\sup \{\hat{k} \in(-\infty, k]: V(x, \hat{k})=0\}$ and $j_{+}(k)=\inf \{\hat{k} \in[k, \infty): V(x, \hat{k})=0\}$, and as before $\sup \emptyset=-\infty$ and $\inf \emptyset=+\infty$. It is straightforward to show that the intrinsic external rate can be viewed as the product of a ratcheting procedure, so that in analogy to Prop. 3 one obtains $\psi(\psi(\psi(\cdots \psi(k) \cdots))) \rightarrow j(k)$. Finally, as in Prop. 4 $j(k)$ can be used as a decision criterion where the relevant direction of the inequality is analogous to the logic in Remark 4. That is, for any internal rate $k \in \mathbb{R}, V(x, k) \geq 0$ if and only if $j(k) \leq k$. Fig. 5 illustrates the ratcheting procedure, which is the dual equivalent of the process depicted in Fig. 4. Note that $j(k)$ has values in $\overline{\mathcal{I}}(x)$ but that the stability properties of the elements of $\mathcal{I}(x)$ are essentially reversed ${ }^{22}$ Intuitively, an intrinsic

\footnotetext{
${ }^{22}$ In the differentiable case (with strict monotonicity, see also footnote 19), this follows from the inverse function theorem (Rudin 1976, pp. 221/223), since $\dot{\psi}(k) \equiv 1 / \dot{\varphi}(\psi(k))$.
} 


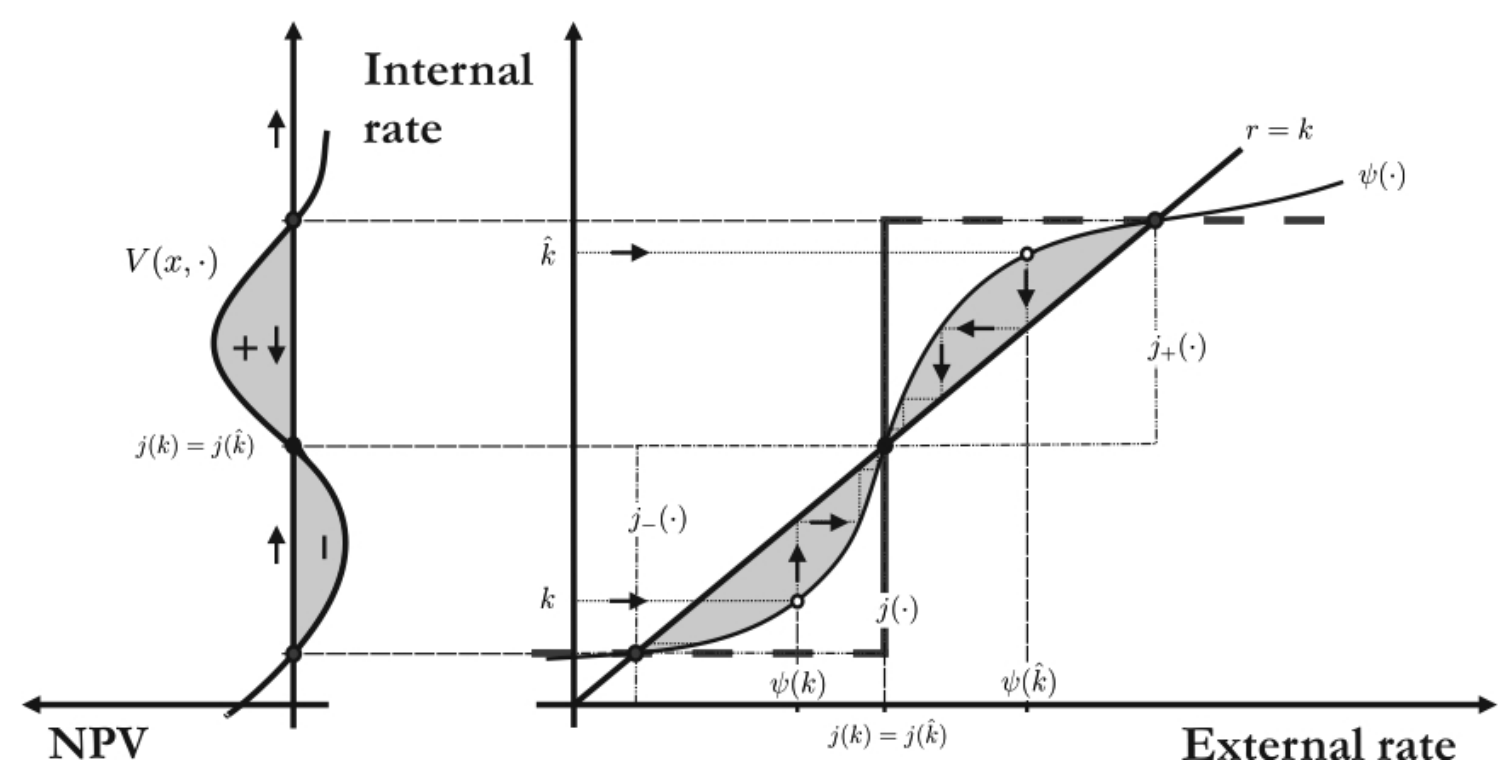

Figure 5: Computation of the intrinsic external rate.

external rate does one of two things based on given PFR $\psi(k)$ : for a profitable project, it presents the fully ratcheted-down external rate lowering the project value towards zero; for an unprofitable project, it is the fully ratcheted-up external rate raising the project value towards its break-even point.

Remark 9. Similar to Remark 5 after Prop. 1, Prop. 4 can be compactly stated as follows: $(i(r)-r) V(x, r)>0$ for all $r \notin \mathcal{I}(x)$ (resp., $(j(k)-k) V(x, k)<0$ for all $k \notin \mathcal{I}(x))$. The result implies in particular that $V(x, r)>0 \Leftrightarrow i(r)>r$ (resp., $V(x, k) \Leftrightarrow j(k)<k$ ).

Example 14. The intrinsic external rate for the discrete cash-flow stream in Ex. 10 is ${ }^{23}$

$$
j[\bar{k}]= \begin{cases}1 / 4, & \text { if } \bar{k} \in(-1,4), \\ 4, & \text { if } \bar{k}=4, \\ \infty, & \text { if } \bar{k}>4,\end{cases}
$$

for any internal rate $\bar{k}>-1$. Similar to the discussion in Remark 4 after Prop. 1, the investment criterion of Prop. 4 can be equivalently reformulated as $V[\mathbf{x}, \bar{k}] \geq 0$ if and only if $j[\bar{k}] \leq k$. Because of the inbuilt ratcheting ${ }^{24}$ for any profitable investment project the internal reference rate must exceed the intrinsic external rate.

Remark 10 (Scaling Properties). Let $x$ and $\hat{x}$ be two cash-flow streams on $[0, T]$ so that $\hat{x}=\mu x$ for some $\mu \neq 0$, and let $y$ and $\hat{y}$ denote the respective project-balance functions. Then the following scaling (or homogeneity) properties hold: (i) $V(\hat{x}, \cdot)=$ $\mu V(x, \cdot)$; (ii) $\mathcal{I}(\hat{x})=\mathcal{I}(x)$, (iii) If $\mu>0, \hat{y}(t, k, r) \equiv y(t, k, r), \varphi_{\hat{x}}(r) \equiv \varphi_{x}(r)$, and $i_{\hat{x}}(r) \equiv$ $i_{x}(r)$; (iv) If $\mu<0, \hat{y}(t, k, r) \equiv \mu y(t, r, k), \varphi_{\hat{x}}(r) \equiv \psi_{x}(r)$, and $i_{\hat{x}}(r) \equiv i_{-x}(r)$. The proofs for these properties are straightforward and therefore omitted. Property (iv), which results from $f(\mu y, k, r)=\mu f(y, r, k)$ for $\mu<0$, can be best appreciated by the following example.

\footnotetext{
${ }^{23}$ The discrete intrinsic external rate $j[\cdot]$ is obtained (using discrete-time equivalents $j_{ \pm}[\cdot]$ and $\psi[\cdot]$ ) using a procedure analogous to Remark 6 for the discrete version of the selective IRR.

${ }^{24}$ The ratcheting process is such that $k_{0}=k$ and $\psi\left(k_{n}\right)=k_{n-1}$ for $n \geq 1$.
} 
Properties (i) through (iv) in Remark 10 describe how the performance measures behave when the investment projects are scaled (for $\mu>0$ ) or when the perspective shifts to a counterparty (for $\mu<0$ ) that views payables as receivables and vice-versa.

Example 15. Consider the discrete cash-flow stream $x$ as in Ex. 4 and $\hat{x}=-x$ with associated cash-flow vector $\hat{\mathbf{x}}=(16,-100,100)$. Then $\hat{y}_{T}=-y_{T}$, and consequently $\varphi_{\hat{x}}[\bar{r}]=(1+\bar{r}) /(5.25-\bar{r})$ which directly corresponds to $\psi[\cdot]$ for $x$ as computed in Ex. 4. While the set of possible IRRs is invariant to scaling the cash-flow stream with any nonzero factor, in the computation of the selective IRR the stability properties of the fixed points of $\varphi_{\hat{x}}(\cdot)$ are reversed when $\mu<0$, so

$$
i_{\hat{x}}[\bar{r}]= \begin{cases}1 / 4, & \text { if } \bar{r}<4, \\ 4, & \text { if } \bar{r}=4, \\ +\infty, & \text { if } \bar{r}>4,\end{cases}
$$

for any external rate $\bar{r}>-1$.

Remark 11 (Robustness). For a given cash-flow stream $x$, assume that the set $\mathcal{I}(x)$ of potential IRR-values is finite ${ }^{25}$ For any possible external rate $r \in \mathbb{R}$ let $d(r, x)=\{|r-\hat{r}|:$ $\hat{r} \in \mathcal{I}(x)\}$ be the distance of this rate to the closest potential IRR-value. If the external rate $r$ is uncertain in the sense that it can be considered as the realization the random variable $\tilde{r}$ which follows some continuous cumulative distribution function (corresponding to a probability density without atoms), then $\operatorname{Prob}(d(\tilde{r}, x)>0)=1$. Hence, because the selective $i(r)$ can have (finite-size) jumps at most on $\mathcal{I}(x)$, with probability one it will be possible, once the realization $r$ is known, to find a neighborhood $\mathcal{N}_{\varepsilon}=(r-\varepsilon, r+\varepsilon)$ (e.g., with $\varepsilon=d(r, x) / 2)$ such that $i(\hat{r})=i(r)$ for all $\hat{r} \in \mathcal{N}_{\varepsilon}$. In other words, almost surely the selective IRR will be insensitive to small variations of the external rate.

\section{Conclusion}

As mentioned in Sec. 1, the internal rate of return (IRR) is used widely in practice, often because of its direct comparability to asset returns in financial markets. For example, it appears naturally as "yield to maturity" of a fixed-income security, i.e., the rate of return of an interest-bearing investment held to its maturity date at $T$. The well-known problems with the IRR resulting from its generic nonexistence or nonuniqueness disappear by using instead the selective IRR, which always exists and selects, for any external rate $r$, a unique element of the extended set $\overline{\mathcal{I}}(x)$ of possible IRRs (including $\pm \infty$ ) for a given cash-flow stream $x$. The construction of the selective IRR is based on the project investment rate $\varphi(r)$ and therefore inherits its NPV-consistence, in the sense that the difference between $i(r)$ (resp., $\varphi(r))$ and $r$ has the same sign as the NPV of the project.

The selective IRR does not suffer from the drawbacks that plague other rate-of-returnbased criteria such as the mixing inconsistency uncovered in Sec. 3.2. Its computation is simpler than determining the set $\mathcal{I}(x)$ (see Remark 7). It is also arguably more robust than NPV in the following sense (with details in Remark 11): Pick a random nominal rate $r_{0}$; with probability 1 there exists $\varepsilon>0$ such that $i(r)=i\left(r_{0}\right)$ for all $r \in\left(r_{0}-\varepsilon, r_{0}+\varepsilon\right)$. That

\footnotetext{
${ }^{25}$ This holds for any discrete cash-flow stream over a finite time horizon. More generally, it is enough for the robustness argument here if $\mathcal{I}(x)$ is countable.
} 
is, except for a measure-zero set of external rates, the selective IRR will be exact even in the presence of some uncertainty. On the other hand, given a noisy external rate the NPV of a project is generically be inexact (with probability 1), and, because of the nonlinearity of $V(x, r)$ in $r$, it cannot simply be replaced by its expected value. The selective IRR can be used to measure the sensitivity of an investment decision with respect to changes in the external rate, just like the traditional IRR (Berk and DeMarzo 2007, p. 150).

It is useful to note that the selective IRR $i(r)$ is compatible with the standard Keynesian notion of marginal efficiency of capital (Dorfman 1981) and that it does depend on the external rate of return. The latter can be viewed as a public signal that allows the coordination of firm and outside capital, in order to determine an appropriate internal rate of return. More specifically, it is possible to interpret the selective IRR as equilibrium of an alternating-offer bargaining game (without delay between rounds) between two firms and a venture capitalist. The latter operates on a capital market with risk-free rate of return $r_{0}=r$. The two firms have identical investment projects, here assumed profitable and described by the cash-flow stream $x$ on $[0, T]$. They Bertrand-compete on the rent they are willing to provide to the investor on borrowed capital. As a result the firms offer the project investment rate $r_{n+1}=\varphi\left(r_{n}\right)$, based on the last offer $r_{n}$ of the venture capitalist who in turn responds by asking for that maximal rate, after which the firms revise their offers, and so forth, until they arrive at the selective IRR $i(r)=\lim _{n \rightarrow \infty} r_{n}$ as the limit of this ratcheting procedure, further described in the proof of Prop. 3. The initial rate $r$ therefore serves as a coordination device for the selection of a Nash equilibrium in a set of equilibria which (via bijection) directly corresponds to the extended set $\overline{\mathcal{I}}(x)$ of possible selective IRRs, defined after Eq. (10). In this context, the selective IRR behaves in analogy to an (ideal) equilibrium refinement which always produces a unique value. ${ }^{26}$

The well-known drawbacks of the NPV rule (Ross 1995; footnote 17) apply also to the (by Prop. 4 equivalent) selective-IRR criterion. However, in the absence of interestrate uncertainty those drawbacks can be eliminated by solving all stochastic optimization problems (e.g., related to the optimal stopping problem to determine the timing of the investment) and then applying the NPV criterion or the selective-IRR criterion to the resulting expected cash-flow stream. The two investment criteria should be viewed as complementary, since cash-flow streams of the same NPV can have dramatically different returns ${ }^{27}$ When it comes to choosing between the two criteria, scalability of an investment opportunity is the key. If the investment is not scalable, then for a given external rate the NPV provides a notion of its present value, while the selective IRR fails to capture the size of the gain. If it is, then the selective IRR provides a notion of the achievable return on capital invested, not reflected in the NPV.

\footnotetext{
${ }^{26}$ Kreps (1990, Ch. 5) points to equilibrium multiplicity as one of the major drawbacks of game theory and consequently to equilibrium refinement as a potential saviour. However, the issues with IRR as investment criterion are even more serious than equilibrium multiplicity is for games, since $\mathcal{I}(x)$ not only might not be a singleton, but might even be empty. By contrast the selective IRR always exists and is unique as an element of $\overline{\mathcal{I}}(x)=\mathcal{I}(x) \cup\{-\infty, \infty\}$.

${ }^{27}$ For example, given a fixed external rate $\bar{r}>-1$ the discrete cash-flow stream $x(t)$ on $[0,1]$, represented by the vector $\mathbf{x}=\left(-\frac{1+\bar{r}}{k-\bar{r}}, \frac{1+\bar{k}+\bar{r}+\bar{k} \bar{r}}{\bar{k}-\bar{r}}\right)$, satisfies the "isoperimetric constraint" of having the constant value $V[\mathbf{x}, \bar{r}]=1$ while at the same time the selective IRR is $i[\bar{r}]=\bar{k}$, for any $\bar{k}>\bar{r}>-1$.
} 


\section{Acknowledgements}

The author would like to thank Naveed Chehrazi, Rüdiger Fahlenbrach, Damir Filipovich, Julien Hugonnier, and participants of the Brownbag Seminar at the Swiss Finance Institute for helpful comments and suggestions.

\section{Appendix A: NPV-Consistency}

Let $x$ be a cash-flow stream on $[0, T]$. As alluded to in Sec. 1.1, a return criterion $\rho(r)$ is termed $N P V$-consistent if the excess return $\alpha=\rho(r)-r$ has the same sign as the net present value of $x$ for any external rate $r$, i.e. ${ }^{28}$

$$
(\rho(r)-r) V(x, r)>0 \quad \forall r \notin \mathcal{I}(x) .
$$

The definition of NPV-consistency in Eq. 16) guarantees that investment decisions based on a mere comparison of the return criterion $\rho(r)$ with the external rate $r$ yields identical results as the classical NPV-rule. By virtue of Remark 5 (after Prop. 1) and Remark 9 (after Prop. 4) the project investment rate (PIR) $\varphi(\cdot)$ and the selective IRR $i(\cdot)$ are NPV-consistent return criteria, in the sense that Eq. (16) holds for all $\rho(\cdot) \in\{\varphi(\cdot), i(\cdot)\}$.

The product approaches by Hazen (2003) and Magni (2010) represent the NPV as the product of the excess return $\alpha$ and the present value of some invested capital, related to the project balance by Teichroew et al. (1965). Below we formulate these approaches in continuous time generalizing the first, and then compare both to PIR and selective IRR.

\section{A.1 Factorization of NPV With Given IRR}

Hazen (2003) uses the concept of an investment stream (Lohmann 1988), defined relative to a given IRR, to formulate an NPV-equivalent investment rule. Specifically, Hazen (2003, p. 35) introduces the notion that a discrete investment stream $c$ with cashflow vector $\mathbf{c}=\left(c_{-1}, \ldots, c_{T}\right)$ yields a (discrete) cash-flow stream $x$ with cash-flow vector $\mathbf{x}=\left(x_{0}, \ldots, x_{T}\right)$ at return $k \in \mathcal{I}[\mathbf{x}]$ if the following three relations are satisfied,

$$
\begin{aligned}
x_{0} & =k c_{-\delta}-\frac{c_{0}-c_{-1}}{\delta}, \\
x_{t} & =k c_{t-\delta}-\frac{c_{t}-c_{t-\delta}}{\delta}, \quad \forall t \in\{1, \ldots, T-1\}, \\
x_{T} & =k c_{T-\delta}-\frac{c_{T}-c_{T-\delta}}{\delta},
\end{aligned}
$$

where $c_{-1}=c_{T}=0$ and $\delta=1$ in Hazen's paper 29 First, if we split the time interval $[-\delta, T]$ for $\delta=T / N$ in $N+1$ intervals, $[-\delta+i \delta,-\delta+(i+1) \delta]$ for $i \in\{0, \ldots, N\}$, then Eqs. (17) -19 translate to

$$
x_{t}=k c_{t-\delta}-\frac{c_{t}-c_{t-\delta}}{\delta}, \quad \forall t \in\{\tau: \tau=-\delta+i \delta, i \in\{0, \ldots, N+1\}, \delta=T / N\},
$$

\footnotetext{
${ }^{28}$ To keep the exposition simple, we restrict attention to the case where the external rate $r$ is given. The situation is (anti-)symmetric when the internal rate $k$ is assumed given, e.g., with the inequality in Eq. (16) reversed.

${ }^{29}$ Hazen's investment stream $\left(c_{0}, \ldots, c_{T-1}\right)$ omits the zero boundary elements $c_{-1}$ and $c_{T}$. The latter carry no extra information, except that they make it possible to combine Eqs. (17)-(19) into Eq. 20).
} 


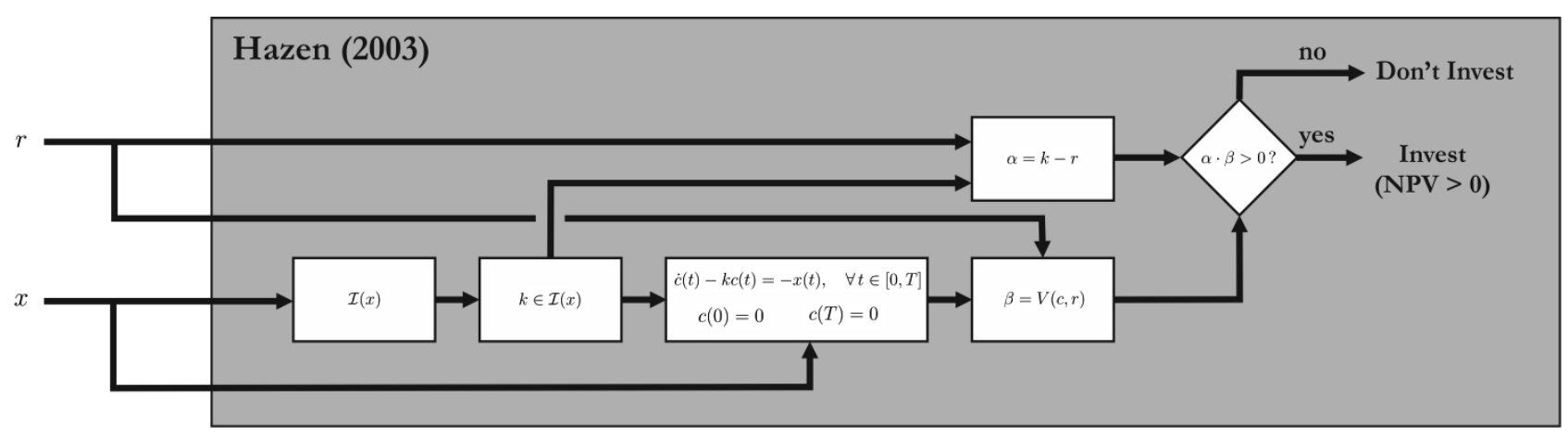

Figure 6: Product approach by Hazen (2003).

where $c_{-\delta}=c_{T}=0$. For $N \rightarrow \infty$ and thus $\delta \downarrow 0^{+}$, Eq. 20 becomes

$$
x(t)=k c(t)-\dot{c}(t), \quad \forall t \in[0, T],
$$

with the boundary conditions $c(0)=0$ and $c(T)=0$. In our continuous-time setting, one obtains the linear initial value problem (IVP),

$$
\dot{c}(t)-k c(t)=-x(t), \quad \forall t \in[0, T], \quad c(0)=0,
$$

with an additional endpoint condition $c(T)=0$. The unique solution of the IVP 22 is obtained via the Cauchy formula (see, e.g., Weber 2011, p. 25),

$$
c(t)=-e^{k t} \int_{0}^{t} e^{-k s} x(s) d s .
$$

Hence, the investment stream $c(t)$ represents the future value, at time $t \in[0, T]$, of the "t-truncated" cash-flow stream $\left.x\right|_{[0, t]}$, defined at time $t$ as $x$ multiplied with the indicator function $\mathbf{1}_{[0, t]}$, i.e., the cash-flow stream $x$ is restricted to be nonzero at most on the interval $[0, t] \cdot 30$ The endpoint condition,

$$
c(T)=-e^{k T} \int_{0}^{T} e^{-k t} x(t) d t=-e^{k T} V(x, k)=0,
$$

amounts to requiring that the present value $V(x, k)$ vanishes, or equivalently, that

$$
k \in \mathcal{I}(x)
$$

i.e., $k$ must be an internal rate of return of the cash-flow stream $x$. From the above it follows that $c$ yields $x$ at rate $k$ if and only if $k \in \mathcal{I}(x)$ and $x(t)=e^{k t} V\left(\left.x\right|_{[0, t]}, k\right)$ for all $t \in[0, T]$. This essentially corresponds to Hazen (2003, Thm. 2), where $c(t)$ is the IRR-implied outstanding capital (at time $t$ ).

\footnotetext{
${ }^{30}$ The investment stream, as solution to the IVP 22 , is closely related to the project balance by Teichroew et al. (1965), as solution to the IVP (3) in Sec. 2 , since $y(t, r, r) \equiv-c(t)$.
} 
Using integration by parts, one finds (taking into account the boundary conditions $c(0)=c(T)=0)$ that 31

$$
\begin{aligned}
V(x, r) & =\int_{0}^{T} e^{-r t} x(t) d t=\int_{0}^{T} e^{-r t}(k c(t)-\dot{c}(t)) d t \\
& =k V(c, r)-\int_{0}^{T} e^{-r t} \dot{c}(t) d t \\
& =k V(c, r)-\left(e^{-r T} c(T)-c(0)-\int_{0}^{T}(-r) e^{-r t} c(t) d t\right) \\
& =(k-r) V(c, r) .
\end{aligned}
$$

Hence, if c yields $x$ at rate $k$, then

$$
V(x, r)=(k-r) V(c, r)
$$

for any rate $r$. This corresponds to Hazen (2003, Thm. 1), where the factor $\alpha=k-r$ is the "excess return" and $\beta=V(c, r)$ the "invested capital." Thus, the present value of the cash-flow stream $x$ can be expressed as a product,

$$
V(x, r)=\alpha \cdot \beta \text {. }
$$

The main result (Hazen 2003, Thm. 4, parts (i) and (ii)) is that $V(x, c)>0$ if and only if $\alpha$ and $\beta$ have the same definite sign. The case where $\beta>0$ is called "net investment" and the case where $\beta<0$ is called "net borrowing". We note that both $\alpha$ and $\beta$ depend on $k$ and $r$, and that $\beta$ also depends on the entire cash-flow stream $x$. Fig. 6 provides an overview of the product approach by Hazen (2003) for reaching a decision about whether to invest in a project with cash-flow stream $x$ at the given external rate $r$.

\section{A.2 Factorization of NPV With Given Investment Stream}

Stated in continuous time, Magni (2010) allows for the choice of an absolutely continuous investment stream $c(t), t \in[0, T]$, of nonzero NPV, and introduces a return-on-investment stream $R=\dot{c}+x$ and a return function $K(t)=R(t) / c(t)$, which is defined as long as $c(t) \neq 0$. With this, one obtains

$$
V(x, r)=V(R, r)-r V(c, r)=(\bar{k}(c, r)-r) V(c, r)
$$

using the "Chisini mean" (Chisini 1929, p. 108; Magni 2010, p. 159)

$$
\bar{k}(c, r)=\frac{V(R, r)}{V(c, r)}=\frac{V(K c, r)}{V(c, r)},
$$

provided that $V(c, r) \neq 0$ by assumption. The value $\bar{k}(c, r)$ is referred to as the "average internal rate of return" (AIRR). It depends on the external rate $r$ and the chosen investment stream $c$. In the definition of the AIRR there is the issue that $K(t)$ is not defined whenever $c(t)=0$, which is briefly mentioned by Magni (2010, Remark 1). Yet it turns

\footnotetext{
${ }^{31}$ This factorization was introduced (in discrete time) by Lohmann (1988, p. 321).
} 
out that it only matters that the NPV $\beta=V(c, r)$ of the chosen investment stream $c$ at the external rate $r$ is nonzero. Eq. $(* * *)$ is of the same form as Eq. $\left.{ }^{* *}\right)$ earlier, with excess return $\alpha=\bar{k}(c, r)-r$ and $\beta=V(c, r)$, where $c$ is given. Because $\beta$ (assumed nonzero) is fixed by the choice of $c$ and Eq. (**) links $\alpha, \beta$ and $V(x, r)$, Magni (2010, p. 160) points out that therefore one needs to set

$$
\alpha=\frac{V(x, r)}{\beta},
$$

for the factorization in Eq. (**) to hold. As in Sec. A.1, the main result (Magni 2010, Thm. 2, parts (i) and (ii)) is that $V(x, c)>0$ if and only if $\alpha$ and $\beta$ have the same definite sign. The factors $\alpha$ and $\beta$ depend on $r$ and the chosen investment stream $c$. Fig. 7(b) provides an overview of the product approach by Magni (2010), termed below "approach $B$," for evaluating the cash-flow stream $x$ at the external rate $r$.

\section{A.3 Comparison to NPV-Consistent Return Criteria}

We now compare the product approaches outlined earlier to PIR and selective IRR as investment criteria in terms of NPV-consistency.

Approach A. By generalizing the product approach by Hazen (2003) in Sec. A.2, we now show that it is possible to fix $\alpha$ in Eq. (**) to an arbitrary nonzero value, which we refer to as "approach A." For this, consider any absolutely continuous investment stream $c$ on $[0, T]$ which satisfies an initial condition of the form $c(0)=c_{0}$, where the constant $c_{0}$ is chosen such that $c(T)=e^{r T} c_{0}$ in view of Eq. (23). With this, the unique solution to the IVP (22) becomes (via the Cauchy formula):

$$
c(t)=\left(c_{0}-\int_{0}^{t} e^{-k s} x(s) d s\right) e^{k t}, \quad \forall t \in[0, T] .
$$

Hence, at $t=T$ the investment stream satisfies

$$
c(T)=\left(c_{0}-\int_{0}^{T} e^{-k t} x(t) d t\right) e^{k T}=\left(c_{0}-V(x, k)\right) e^{k T},
$$

or equivalently,

$$
\left(e^{k T}-e^{r T}\right) c_{0}=e^{k T} V(x, k) .
$$

The interpretation of this condition is that the future value of the project at rate $k$ (with cash-flow stream $x$ ) is equal to what the initial value $c_{0}$ of the investment stream would pay at rate $k$ (in the future, at time $T$ ) if it was borrowed at rate $r$.

Hence, as long as $k \neq r$, the investment stream

$$
c(t)=\left(\frac{V(x, k)}{1-e^{-(k-r) T}}-\int_{0}^{t} e^{-k s} x(s) d s\right) e^{k t}, \quad \forall t \in[0, T],
$$

by Eq. (23) also satisfies Eq. (*), i.e.,

$$
V(x, r)=(k-r) V(c, r) .
$$




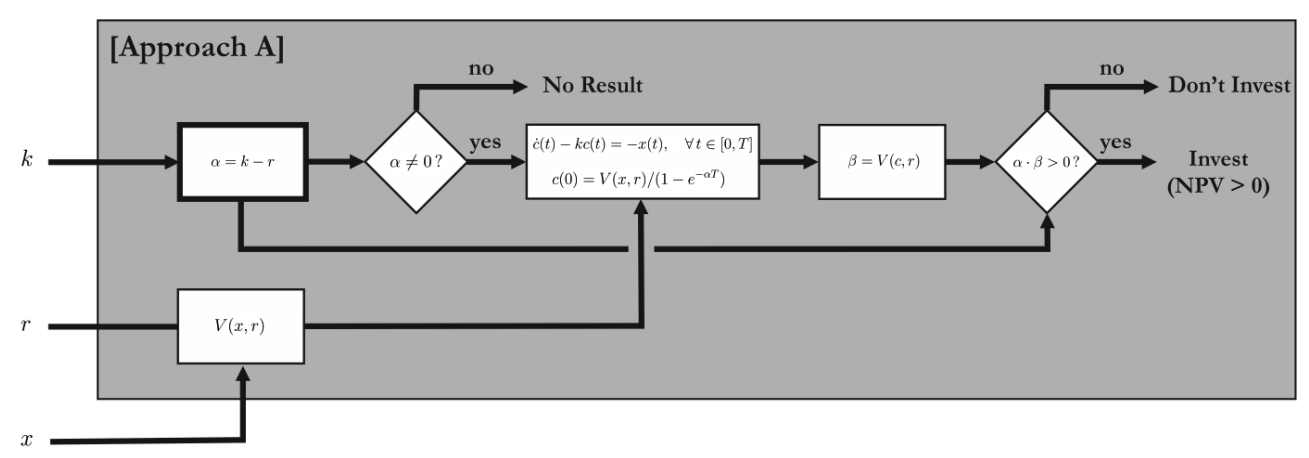

(a)

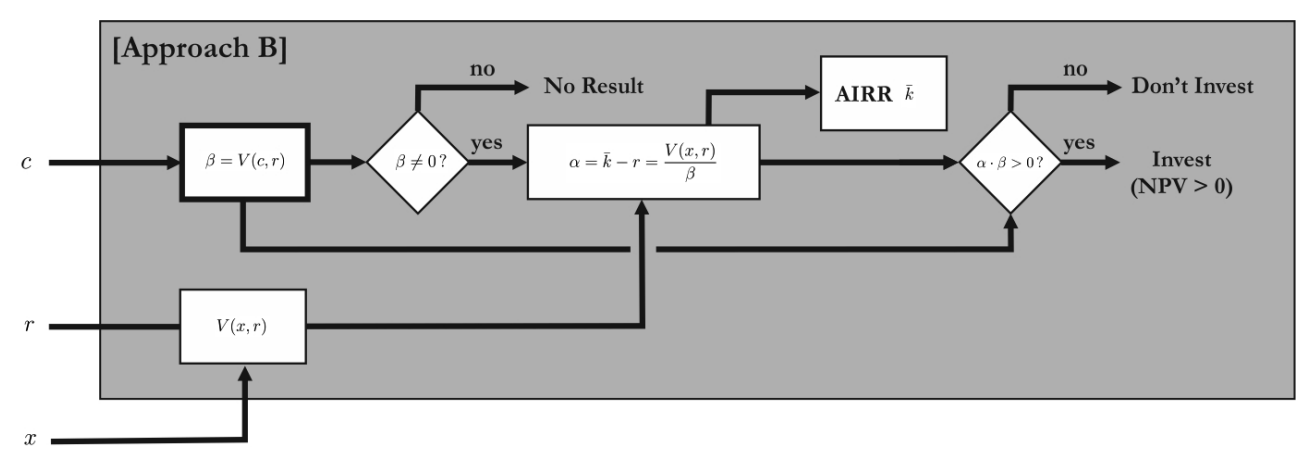

(b)

Figure 7: Product approaches for $V(x, r)=\alpha \cdot \beta$ : (a) fixing $\alpha$; (b) fixing $\beta$.

For this now arbitrary rate $k \neq r$ the same investment rule applies as in Hazen (2003, Thm. 4, parts (i) and (ii)). In the terminology of Eq. $\left(^{* *}\right)$ this amounts to saying that if we fix an arbitrary $\alpha \neq 0$ and

$$
V(x, r)=\alpha \cdot \beta,
$$

then $\beta=V(x, r) / \alpha=V(c, r)$ and we should invest if $\alpha \cdot \beta>0$. It is not possible to glean additional information from the constant $k$, other than that it is different from the known external rate $r$ and that the investment stream $c$ has been chosen as a function of $r$ and $k$ so as to be compatible with $x{ }^{32}$

Approach $B$. Just as approach $A$ fixes $\alpha \neq 0$ in Eq. $\left({ }^{* *}\right)$, the product approach by Magni (2010), referred to as "approach $B$," fixes $\beta \neq 0$ through the choice of an investment stream $c$. In the case where $\beta=V(c, r)=0$ nothing can be concluded because the AIRR is not well-defined as a return criterion. The use of approach $B$ to evaluate a given cash-flow stream $x$ is summarized in Fig. 7(b).

Comparison to NPV-consistent return criteria. So far, we have shown that it is possible, by either one of the two product approaches, to represent the NPV $V(x, r)$ of the cashflow stream $x$ as a product of two factors as in Eq. (**), in terms of $\alpha$ (excess return) and $\beta$ (value of outstanding capital), choosing one of the factors which then implies the

\footnotetext{
${ }^{32}$ The case where $k=r$, or equivalently $\alpha=0$, deserves special attention. Since $x$ and $r$ are fixed, $V(x, r)$ can in general be nonzero. When $k=r$, Eq. (**) cannot possibly hold as long as $\beta$ is finite. Moreover, as $k \rightarrow r$ the investment stream becomes unbounded, i.e., $|\beta|=|V(c, r)| \rightarrow \infty$ as $k \rightarrow r$. In particular, nothing about the sign of $V(x, r)$ can be concluded in that case.
} 


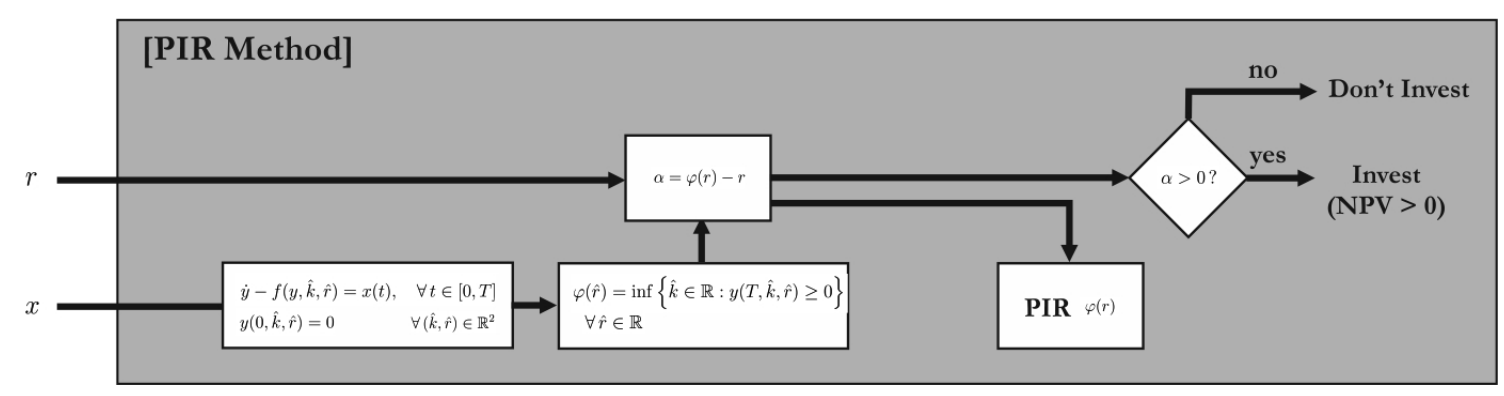

(a)

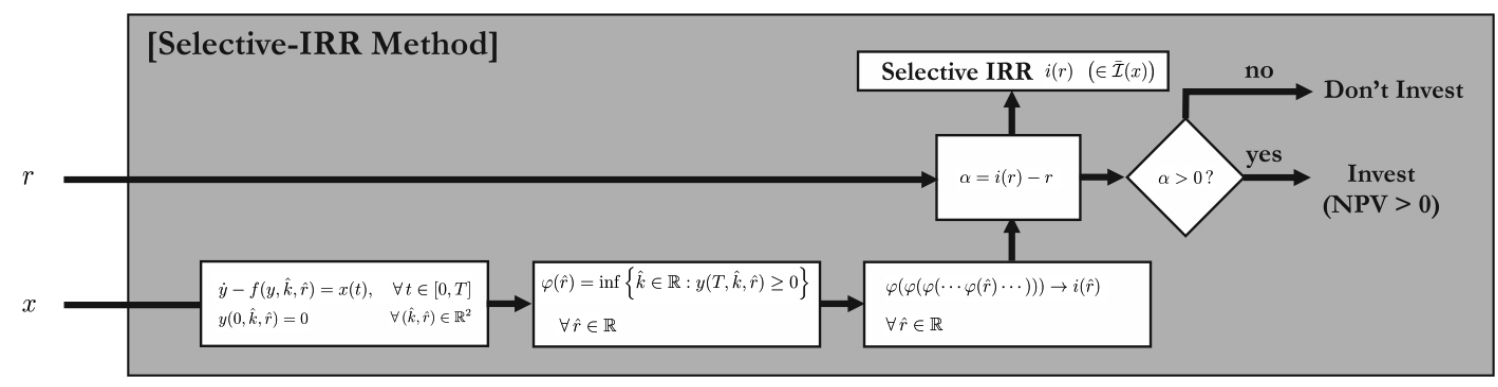

(b)

Figure 8: NPV-consistent return criteria: (a) PIR; (b) selective IRR.

other factor. There may be practical reasons to suggest fixing one factor. For example, Hazen (2003) by requiring that $k$ be an internal rate of return of $x$, it is $V(x, k)=0$ and with $c_{0}=0$ satisfies condition (24) for any $r$, so the investment stream $c$ becomes independent of $r$. The interpretation of an investment stream for each element $k \in \mathcal{I}(x)$ may add some complementary insights; it effectively considers $\alpha, \beta$ pairs whose product is constant. Similarly, Magni (2010) allows for any investment stream, which in some situations may be practically observed as invested capital accompanying the cash-flow stream $x$. Thus fixes $\beta=V(c, r)$, which implies an excess return $\alpha=V(x, r) / \beta$, provided $\beta \neq 0$. While both approaches therefore add some flexibility to the interpretation of project return, they lack intrinsic guidance about which return characterizes the cashflow stream $x$ in the absence of additional arguments and information.

The product approaches are in general not NPV-consistent in the sense of Eq. (16): in approach $A, \alpha \neq 0$ is arbitrary and therefore generally of a different sign than $V(x, r)$; in approach $B, \beta \neq 0$ is arbitrary, so that $\alpha=V(x, r) / \beta$ is a of different sign than $V(x, r)$ whenever $\beta=V(c, r)<0$. Hence, unlike PIR and selective IRR (see Fig. 8), in the absence of any additional information, the sign of the excess return $\alpha$ in the product approaches cannot be used as a decision criterion 33

\footnotetext{
${ }^{33}$ Since one of the factors in Eq. $\left({ }^{* *}\right)$ is chosen freely (nonzero) using heuristics, the remaining factor must carry all the decision-relevant information. Moreover, computing the remaining factor generally involves computing the NPV as a subtask. For example, under approach $B$, instead of the investment stream $c$ (a function) one can just choose any $\beta \neq 0$ (a constant, which defines an equivalence class of investment streams), compute the "excess return" $\alpha=V(x, r) / \beta$, and then decide to invest in a project with cash-flow stream $x$ if $\alpha \cdot \beta>0$. Yet, for the mere purpose of deciding whether $x$ presents an attractive investment opportunity (or not) it would be even more straightforward (involving strictly less computation) to determine the sign of $V(x, r)$ directly.
} 


\section{References}

[1] Alchian, A.A. (1955) "The Rate of Interest, Fisher's Rate of Return Over Costs, and Keynes' Internal Rate of Return," American Economic Review, Vol. 45, No. 5, pp. $938-943$.

[2] Altshuler, D., Magni, C.A. (2012) "Why IRR is Not the Rate of Return on Your Investment: Introducing the AIRR to the Real Estate Community," Journal of Real Estate Portfolio Management, Vol. 18, No. 218, pp. 219-230.

[3] Arrow, K.J., Levhari, D. (1969) "Uniqueness of the Internal Rate of Return with Variable Life Investment," Economic Journal, Vol. 79, No. 315, pp. 560-566.

[4] Aubin, J.-P. (1977) Applied Abstract Analysis, Wiley, New York, NY.

[5] Berk, J., DeMarzo, P. (2007) Corporate Finance, Pearson/Addison Wesley, New York, NY.

[6] Berkovitch, E., Israel, R. (2004) "Why the NPV Criterion Does Not Maximize NPV," Review of Financial Studies, Vol. 17, No. 1, pp. 239-255.

[7] Biondi, Y. (2006) "The Double Emergence of the Modified Internal Rate of Return: The Neglected Financial Work of Duvillard," European Journal of the History of Economic Thought, Vol. 13, No. 3, pp. 311-335.

[8] BöHm-BAwerk, E. Von (1889) Positive Theorie des Kapitales, Wagner, Innsbruck, Austria.

[9] Bodie, Z., Kane, A., Marcus, A.J. (1996) Investments, 3rd Ed., McGraw-Hill, New York, NY.

[10] Brealey, R.A., Myers, S.C. (1991) Principles of Corporate Finance, 4th Ed., McGraw-Hill, New York, NY.

[11] Brounen, D., De Jong, A., KoediJk, K. (2004) "Corporate Finance in Europe: Confronting Theory with Practice," Financial Management, Vol. 33, No. 4, pp. 71101.

[12] Chisini, O. (1929) "Sul Concetto di Media," Periodico di Matematiche, Ser. 4, Vol. 9, No. 4, pp. 106-116.

[13] Dixit, A.K. (1992) "Investment and Hysteresis," Journal of Economic Perspectives, Vol. 6, No. 1, pp. 107-132.

[14] Dorfman, R. (1981) "The Meaning of Internal Rates of Return," Journal of Finance, Vol. 36, No. 5, pp. 1011-1021.

[15] Dowell, M., Jarratt, P. (1972) "The "Pegasus" Method for Computing the Root of an Equation," BIT Numerical Mathematics, Vol. 12, No. 4, pp. 503-508.

[16] DuVillard, E.E. (1787) Recherches sur les Rentes, les Emprunts et les Remboursemen $[t] s$, Imprimeur-Libraire François Dufart, Geneva, Switzerland.

[17] Downes, J., Goodman, J.E. (1998) Dictionary of Finance and Investment Terms, 5th Ed., Barron's Educational Series, Hauppauge, NY.

[18] Fisher, I. (1930) The Theory of Interest, Macmillan, New York, NY.

[19] Flemming, J.S., Wright, J.F. (1971) "Uniqueness of the Internal Rate of Return: A Generalisation," Economic Journal, Vol. 81, No. 322, pp. 256-263. 
[20] Gioja, M. (1817) Nuovo Prospetto Delle Scienze Economiche (Tome Sesto ed Ultimo), Presso Giorgio Pirotta, Milan, Italy.

[21] Graham, J.R., Harvey, C.R. (2001) "The Theory and Practice of Corporate Finance: Evidence from the Field," Journal of Financial Economics, Vol. 60, No. 23, pp. 187-243.

[22] Hartman, J.C., Schafrick, I.C. (2004) "The Relevant Internal Rate of Return," Engineering Economist, Vol. 49, No. 2, pp. 139-158.

[23] Hazen, G.B. (2003) "A New Perspective on Multiple Internal Rates of Return," Engineering Economist, Vol. 48, No. 1, pp. 31-51.

[24] Hildreth, C. (1946) "A Note on Maximization Criteria," Quarterly Journal of Economics, Vol. 61, No. 1, pp. 156-164.

[25] Hirshleifer, J. (1958) "On the Theory of Optimal Investment Decision," Journal of Political Economy, Vol. 66, No. 4, pp. 329-352.

[26] Ingersoll, J.E., Ross, S.A. (1992) "Waiting to Invest: Investment and Uncertainty," Journal of Business, Vol. 65, No. 1, pp. 1-29.

[27] Keynes, J.M. (1936) The General Theory of Employment, Interest, and Money, Macmillan, New York, NY.

[28] Kreps, D.M. (1990) Game Theory and Economic Modeling, Clarendon Press, Oxford, United Kingdom.

[29] Lang, S. (1993) Real and Functional Analysis, 3rd Ed., Springer, New York, NY.

[30] Lohmann, J.R. (1988) "The IRR, NPV and the Fallacy of the Reinvestment Rate Assumptions," Engineering Economist, Vol. 33, No. 4, pp. 303-330.

[31] Lorie, J.H., Savage, L.J. (1955) "The Problems in Rationing Capital," Journal of Business, Vol. 28, No. 4, pp. 229-239.

[32] Magni, C.A. (2009) "Investment Decisions, Net Present Value and Bounded Rationality," Quantitative Finance, Vol. 9, No. 8, pp. 967-979.

[33] Magni, C.A. (2010) "Average Internal Rate of Return and Investment Decisions: A New Perspective," Engineering Economist, Vol. 55, No. 2, pp. 150-180.

[34] Magni, C.A. (2013) "The Internal Rate of Return Approach and the AIRR Paradigm: A Refutation and a Corroboration," Engineering Economist, Vol. 58, No. 2, pp. 73-111.

[35] McDonald, R., Siegel, D. (1986) "The Value of Waiting to Invest," Quarterly Journal of Economics, Vol. 101, No. 4, pp. 707-728.

[36] Osborne, M. (2010) “A Resolution to the NPV-IRR Debate?" Quarterly Review of Economics and Finance, Vol. 50, No. 2, pp. 234-239.

[37] Pierru, A. (2010) "The Simple Meaning of Complex Rates of Return," Engineering Economist, Vol. 55, No. 2, pp. 105-117.

[38] Poterba, J.M., Summers, L.H. (1995) "A CEO Survey of U.S. Companies' Time Horizons and Hurdle Rates," Sloan Management Review, Vol. 37, No. 1, pp. 43-53.

[39] Ross, S.A. (1995) "Uses, Abuses, and Alternatives to the Net-Present-Value Rule," Financial Management, Vol. 24, No. 3, pp. 96-102. 
[40] Rudin, W. (1976) Principles of Mathematical Analysis, 3rd Ed., McGraw-Hill, New York, NY.

[41] Samuelson, P.A. (1937) "Some Aspects of the Pure Theory of Capital," Quarterly Journal of Economics, Vol. 51, No. 3, pp. 469-496.

[42] Schall, L.D., Sundem, G.L., Geijsbeek, W.R. (1978) "Survey and Analysis of Capital Budgeting Methods," Journal of Finance, Vol. 33, No. 1, pp. 281-287.

[43] Schwartz, L. (1950) Théorie des Distributions, Hermann, Paris, France.

[44] Scitovsky, T. (1971) Welfare and Competition, Irwin, Homewood, IL.

[45] Stanley, M.T., Block, S.B. (1984) "A Survey of Multinational Capital Budgeting," Financial Review, Vol. 19, No. 1, pp. 36-54.

[46] Teichroew, D., Robichek, A.A., Montalbano, M. (1965) "An Analysis of Criteria for Investment and Financing Decisions under Certainty," Management Science, Vol. 12, No. 3, pp. 151-179.

[47] Weber, T.A. (2011) Optimal Control Theory with Applications in Economics, MIT Press, Cambridge, MA.

[48] Wright, J.F. (1959) "The Marginal Efficiency of Capital," Economic Journal, Vol. 69, No. 276, pp. 813-816. 